THE AstrophysicAL JouRnAL, 567:544-565, 2002 March 1

(C) 2002. The American Astronomical Society. All rights reserved. Printed in U.S.A.

\title{
SIGNATURES OF CONVECTION IN THE SPECTRUM OF PROCYON: FUNDAMENTAL PARAMETERS AND IRON ABUNDANCE
}

\author{
Carlos Allende Prieto \\ McDonald Observatory and Department of Astronomy, University of Texas, RLM 15.308, Austin, TX 78712-1083; callende@hebe.as.utexas.edu \\ MARTIN ASPLUND \\ Uppsala Astronomical Observatory, Box 515, SE-751 20, Uppsala, Sweden; martin@astro.uu.se \\ RAMÓN J. GARCÍA LóPEZ ${ }^{1}$ \\ Instituto de Astrofísica de Canarias, E-38200 La Laguna, Tenerife, Spain; rgl@11.iac.es \\ AND \\ DAVID L. LAMBERT \\ McDonald Observatory and Department of Astronomy, University of Texas, RLM 15.308, Austin, TX 78712-1083; dll@astro.as.utexas.edu \\ Received 2001 September 16; accepted 2001 October 23
}

\begin{abstract}
We have observed the spectrum of Procyon A (F5 IV) from 4559 to $5780 \AA$ with a signal-to-noise ratio of $\sim 10^{3}$ and a resolving power of $2 \times 10^{5}$. We have measured the line bisectors and relative line shifts of a large number of $\mathrm{Fe}$ I and $\mathrm{Fe}$ II lines, comparing them to those found in the solar spectrum. A three-dimensional hydrodynamical model atmosphere has been computed and is tested against observations. The model reproduces in detail most of the features observed, although we identify some room for improvement. At all levels, the comparison of the three-dimensional time-dependent calculations with the observed spectral lines shows a much better agreement than for classical homogeneous models, making it possible to refine previous estimates of the iron abundance, the projected rotational velocity, the limb darkening, and the systemic velocity of the Procyon binary system. The difference between the iron abundance determined with the three-dimensional model and its one-dimensional counterpart is $\lesssim$ 0.05 dex. We find consistency between the iron abundance derived from $\mathrm{Fe} \mathrm{I}$ and $\mathrm{Fe}$ II lines, suggesting that departures from LTE in the formation of the studied lines are relatively small. The scatter in the iron abundance determined from different lines still exceeds the expectations from the uncertainties in the atomic data, pointing out that one or more components in the modeling can be refined further.

Subject headings: convection - line: formation - line: profiles - stars: abundances stars: atmospheres - stars: individual (Procyon A)
\end{abstract}

\section{INTRODUCTION}

Widely available grids of model atmospheres are based on a classical set of assumptions that have remained largely unchanged for many years. In brief, the classical assumptions are that the atmosphere consists of plane-parallel homogeneous layers in hydrostatic and local thermodynamic equilibrium through which a constant (radiative plus convective) flux is transmitted. This is not to say that model atmospheres have not been progressively refined; one notes, for example, the increasing completeness with which line blanketing is included as an opacity source. The geometric assumption of plane-parallel homogeneous layers has been replaced by the adoption of spherically homogeneous layers for construction of model atmospheres of supergiants and giants. More recently, the assumption of local thermodynamic equilibrium (LTE) has been replaced to a limited extent by that of non-LTE (NLTE), largely for models built to represent individual stars. Another classical assumption, hydrostatic equilibrium, has more tenaciously resisted refinement. This paper discusses models that dispense with this assumption (and the geometric assumption of homogeneous layers) by simulating the surface convection as a time-dependent hydrodynamic phenomenon.

The models representing atmospheric convection are a development of three-dimensional hydrodynamical models

\footnotetext{
${ }^{1}$ Departamento de Astrofísica, Universidad de La Laguna, E-38206 La Laguna, Spain.
}

that realistically represent solar granulation (Stein \& Nordlund 1998; Asplund et al. 2000a). Previous extensions of the models to stars have been discussed by Dravins \& Nordlund (1990) (Procyon A, $\alpha$ Cen A and B, and $\beta$ Hyi) and Asplund et al. (1999) (metal-poor stars HD 84937 and HD 140283). In this paper we confront the models with an extensive set of line profiles of Procyon A obtained at very high resolution and high signal-to-noise ratio $(\mathrm{S} / \mathrm{N})$. Our tests are similar to those discussed by Dravins \& Nordlund (1990) but provide a much stiffer challenge of the models.

Crudely, the three-dimensional hydrodynamical model atmosphere comprises areas of hot rising gas (granules) and cool sinking gas (intergranular lanes). Relative to a line profile from a classical atmosphere, the lines are broader, shifted, asymmetric, and of a different equivalent width. Derivation of fundamental parameters, abundances, and other quantities extracted from observed spectra has not been attempted using three-dimensional stellar model atmospheres with a few exceptions (e.g., Bruls \& Rutten 1992; Atroshchenko \& Gadun 1994; Kiselman \& Nordlund 1995; Shchukina \& Trujillo Bueno 2001). This may be a serious omission because modeling the effect of the convective motions and temperature inhomogeneities on the equivalent widths through the parameters known as microand macroturbulence may represent an important source of systematic errors. To the extent that equivalent widths are used to derive defining fundamental parameters, classical and three-dimensional hydrodynamical models will yield different results. For the solar case, however, Allende Prieto 
et al. (2001) have shown that the abundances derived using a numerical inversion based on a one-dimensional model atmosphere are accurate to within 0.04 dex. Naturally, this will not hold in general for other stars and elements, and it is unclear whether the same result is valid for classical, theoretical, one-dimensional models.

Observational scrutiny of surface convection has concentrated on the measurement of the asymmetry of lines, which is generally quantified as a line bisector (Kulander \& Jefferies 1966). Line bisectors have been analyzed in the Sun (e.g., Dravins, Lindegren, \& Nordlund 1981) and stars across the Hertzsprung-Russell diagram (e.g., Gray 1982; Gray \& Toner 1986; Gray \& Nagel 1989). The measurement of line shifts has not received the same attention (e.g., Allende Prieto \& García López 1998; Hamilton \& Lester 1999). We refer the reader to Dravins (1999) for a recent review on line asymmetries and shifts generated by surface convection.

Our goal is to test a new three-dimensional model of Procyon using a large collection of lines observed at high resolution and high $\mathrm{S} / \mathrm{N}$. Tests employ the line shifts, asymmetries, and widths, or in other words, the detailed line profiles. This is the first such comprehensive test of threedimensional hydrodynamical models for a star other than the Sun. All the calculations described in this paper assume LTE.

\section{OBSERVATIONS}

The optical spectrum of Procyon was observed from McDonald Observatory on 1999 January 30 and 31 (UT). We made use of the Harlan J. Smith $2.7 \mathrm{~m}$ telescope and the 2dcoudé spectrograph (Tull et al. 1995), at the focal station F1, delivering an FWHM resolving power of $R \equiv \lambda /$ $\delta \lambda \simeq 2 \times 10^{5}$. Five different spectral setups, selected by tilting the E2 grating, provided almost complete spectral coverage from 4559 to $5780 \AA$, and different exposures (up to 10 for a single setup) were co-added to reach $\mathrm{S} / \mathrm{Ns}$ per pixel in the range 550-2000.

A large number of flat-field exposures were acquired in order to correct the pixel-to-pixel sensitivity pattern (typically smaller than $0.5 \%$ ) without degrading the potentially high $\mathrm{S} / \mathrm{N}$ of the spectra. The individual exposures of Procyon were shorter than, or equal to, 5 minutes to keep the signal within the linear range of the detector and to minimize the blurring due to variations in Earth's motion and instrumental drifts. Standard procedures (within $I A^{2}$ ) were used to remove the bias level and scattered light, to correct the pixel-to-pixel variations of sensitivity, and to extract the spectra. The spectral coverage is complete, except for 10 gaps spanning from 0.2 to $12.5 \AA$ wide. For each spectral setup, $\sim 100 \mathrm{Th}-\mathrm{Ar}$ emission lines were used to define the wavelength scale, by fitting a twodimensional polynomial with an rms at least 10 times smaller than the dispersion. The flux distribution along any given order was normalized by a polynomial. While the normalization procedure is expected to be adequate in spectral regions where the density of absorption lines is low and the lines present are not very strong, the limited coverage of the individual spectral orders $(\sim 20 \AA)$ precludes an accu-

\footnotetext{
${ }^{2}$ IRAF is distributed by the National Optical Astronomy Observatories, which are operated by the Association of Universities for Research in Astronomy, Inc., under cooperative agreement with the National Science Foundation.
}

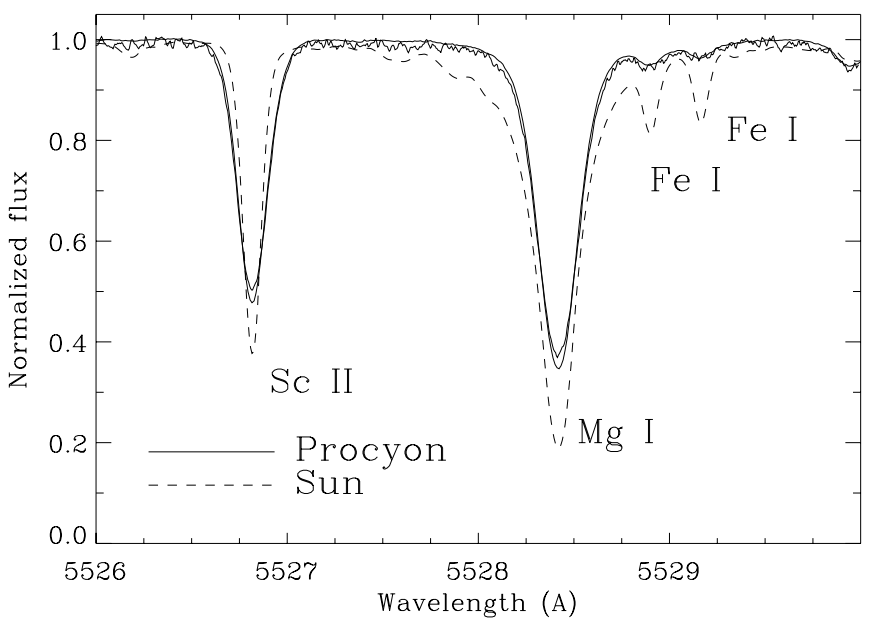

Fig. 1.-Comparison of the McDonald spectrum of Procyon (thick solid line) with the photographic atlas of Griffin \& Griffin (1979; thin solid line) and the solar atlas of Kurucz et al. (1984; thick dashed line).

rate continuum tracing in the proximity of strong lines and in crowded spectral regions.

The multiorder spectra permit a precise correction of the velocity variations and instrumental shifts between different exposures, cross-correlating them order by order. In all cases the standard deviation of the shifts measured between two frames from the 16 different orders was less than $50 \mathrm{~m}$ $\mathrm{s}^{-1}$, with a mean value of $18 \mathrm{~m} \mathrm{~s}^{-1}$ (s.e.m. ${ }^{3} 4.5 \mathrm{~m} \mathrm{~s}^{-1}$ ). For a given spectral setup, we shifted the individual extracted spectra with a single velocity to overlap them on top of the first exposure. Then, the five different spectral setups were reduced to a common scale by computing and correcting the heliocentric velocity shifts produced by Earth's rotation and the motion of the Earth-Moon barycenter around the Sun. The extracted orders were then combined to compose a spectroscopic atlas, which spans the aforementioned spectral range at a variable dispersion of $0.0083 \leq \Delta \lambda \leq 0.0109$ $\AA$ pixel $^{-1}$. Figure 1 displays the spectrum of Procyon in the vicinity of the $\mathrm{Mg}$ I line at $5528.4 \AA$ and compares it with the solar spectrum (Kurucz, Furenlid, \& Brault 1984) and the photographic atlas of Procyon by Griffin \& Griffin (1979). The new data are publicly available on-line. ${ }^{4}$

In a work of this nature, it is very important to check for possible instrumental effects that might distort the stellar line profiles. The 2dcoudé spectrograph is a well-tested instrument, but instrumental drifts have been suspected (see, e.g., Allende Prieto et al. 1999a). Figure 2 shows the variation of the heliocentric correction for the individual exposures with respect to the first (filled circles) and the velocity drifts determined from cross-correlating the individual exposures with the first as a template. A few relatively large drifts (up to $50 \mathrm{~m} \mathrm{~s}^{-1}$ ) are present between consecutive exposures, but slower drifts are also obvious in some cases. Spectra of the Th-Ar hollow cathode were acquired immediately before and after each stellar exposure. The reference wavelengths for the Th-Ar lines used in the calibration correspond always to dry standard air (760 $\mathrm{mmHg}$ and $15^{\circ} \mathrm{C}$ ). The profusion of calibration spectra should prevent large drifts as variations in pressure, tem-

\footnotetext{
${ }^{3}$ Standard error of the mean: $\sigma / N^{1 / 2}$.

${ }^{4} \mathrm{http}: / /$ hebe.as.utexas.edu/procyon.
} 

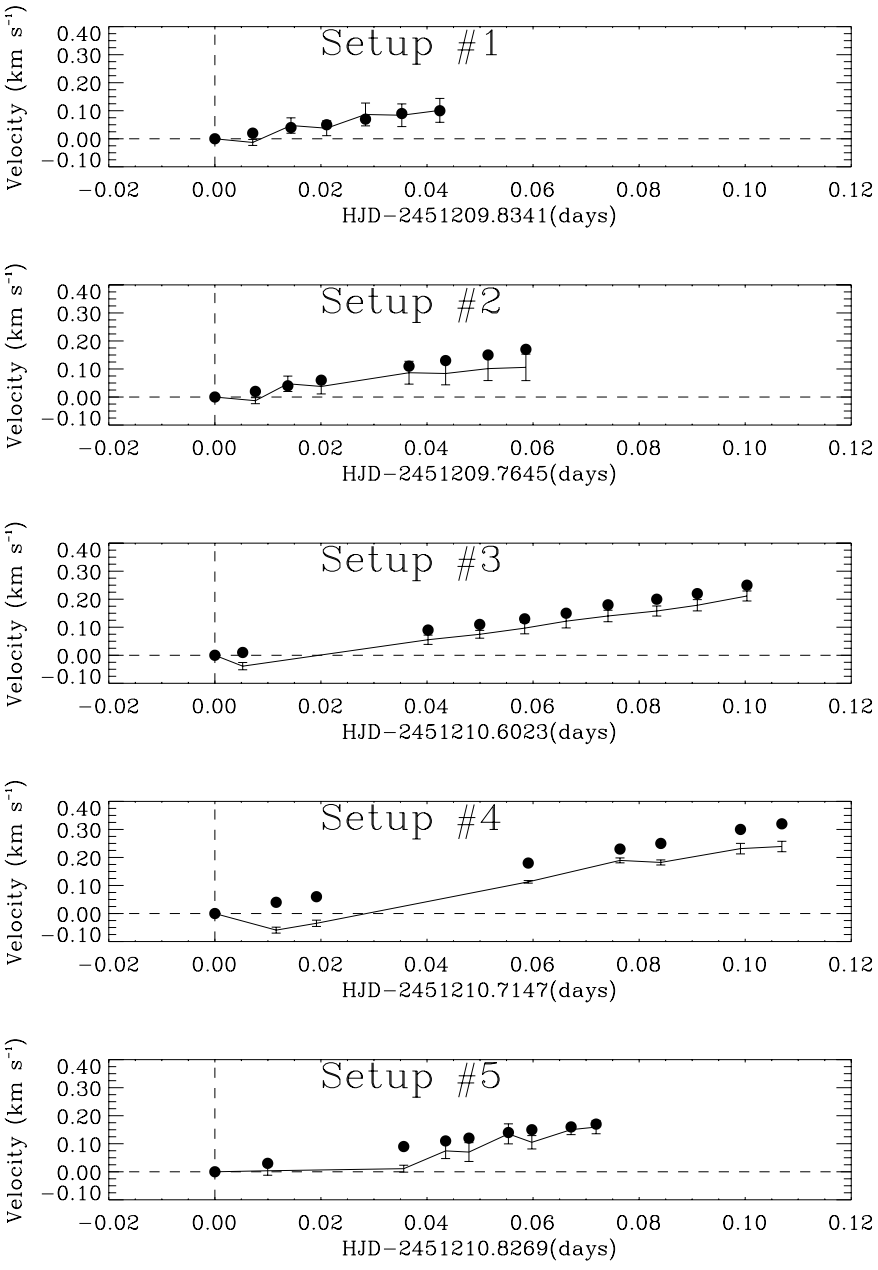

FIG. 2.-Velocity shifts determined from the cross-correlation of the individual exposures for each setup (solid line with error bars). The error bars are determined as the standard error of the mean from the scatter for all the spectral orders. The velocity shifts produced by Earth's motion are marked with filled circles. All shifts are relative to the first spectrum in each series.

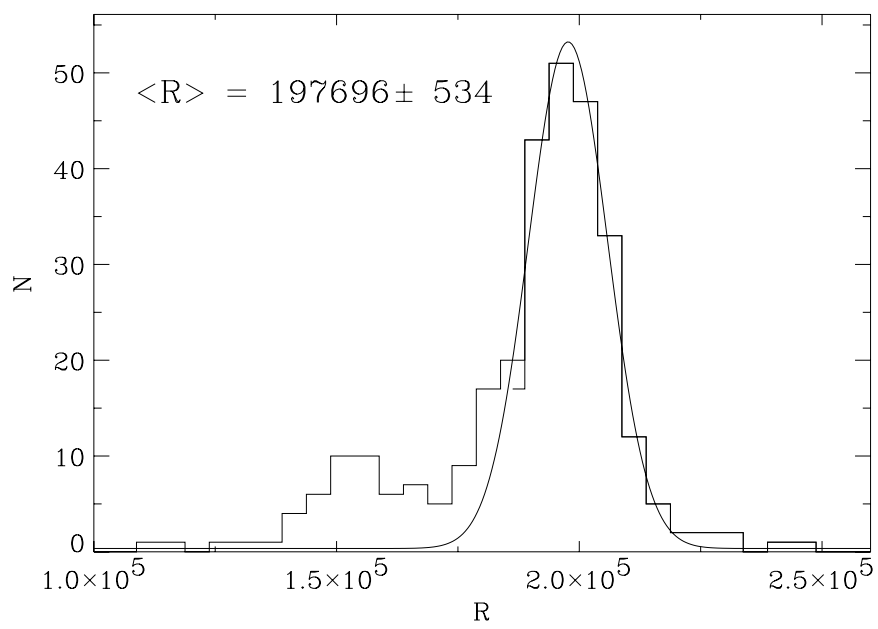

Fig. 3.-Number of emission lines measured in the Th-Ar hollow cathode spectra as a function of the resolving power $R \equiv \lambda / \delta \lambda$. Cosmic rays are easily identified and excluded from the analysis. Systematic errors from blended features are minimized by fitting only the right flank of the nearly Gaussian distribution (thick part of the histogram). perature, and humidity drag the air refraction index. Nonetheless, the sensitivity to changes in temperature, pressure, and humidity is such that variations on small timescales, between the exposures of the Th-Ar hollow cathode and the star, can definitely be a source of small errors. For example, a change of $0.1^{\circ} \mathrm{C}$ from standard conditions would induce a change of $28 \mathrm{~m} \mathrm{~s}^{-1}$, a change of $0.1 \%$ in pressure would induce a drift of $82 \mathrm{~m} \mathrm{~s}^{-1}$, and introducing $0.1 \%$ of water vapor would produce a change of $13 \mathrm{~m} \mathrm{~s}^{-1}$ at $5000 \AA$. Lacking a proper study of these effects in our spectra, we note that changes in pressure within the spectrograph follow in full amplitude those outside the building, and variations as large as $0.07 \% \mathrm{hr}^{-1}$ were measured on the observing dates. Additionally, it is known that the position of the CCD varies with the weight of the $\mathrm{N}_{2}$ Dewar. Differences between how the slit is illuminated by the Th-Ar hollow cathode and the stellar light cannot be ruled out as contributors to systematic errors. Although the crosscorrelation technique makes it possible to determine the shifts among the individual exposures for a given setup to within $\simeq 5 \mathrm{~m} \mathrm{~s}^{-1}$, the observed systematic effects introduce an additional uncertainty in our wavelength calibration, probably not larger than $20-30 \mathrm{~m} \mathrm{~s}^{-1}$. Considering more than 200 lines in the Th-Ar spectra, we confirmed that the nominal resolving power was indeed achieved $(R=197$, $700 \pm 500$; see Fig. 3), with no detectable variation with order or wavelength, but a small dependence on the setup.

\section{OBSERVED SIGNATURES OF CONVECTION}

Different factors may contribute to the observed line asymmetries and shifts. Blends with other features affect particular lines in different ways that cannot be generally predicted with precision. Therefore, it is necessary to average observed line asymmetries and shifts for a large number of lines in order to quantify the signatures of convection with minimal distortion from other shifts. Convective line asymmetries and shifts are closely related and carry complementary information. However, the limited spectral range of earlier works prevented the study of line shifts in depth. In short, stellar studies have ignored line shifts and relied on mean line bisectors computed assuming that the velocity of the lowest end of the bisector is the same for all lines.

Previous studies of line asymmetries in Procyon's spectrum were limited in scope. Gray $(1981 b, 1982)$ analyzed lines in a very small spectral window $(\sim 70 \AA)$ acquired at $R=1.2 \times 10^{5}$. Rice \& Wehlau (1984) attempted the observation but at insufficient spectral resolution. Dravins (1987) analyzed 11 lines using the ESO coudé spectrometer double-pass photoelectric scanner at $R=2 \times 10^{5}$, and he strengthened his results by demonstrating that, despite limited $\mathrm{S} / \mathrm{N}$, the scanned version of the photographic atlas of Griffin \& Griffin (1979) could be used to derive statistically meaningful averaged bisectors for sets of lines with different strengths. Our much larger spectral coverage than previous CCD studies, higher $\mathrm{S} / \mathrm{N}$ and resolution, and thorough control of possible systematic effects set the stage for the first detailed study of asymmetries and shifts for a large sample of iron lines. The gravitational redshift and the orbital and radial velocities affect the zero point of the velocity scale, but the information we are searching for is contained in the line bisectors and their relative displacements.

To minimize systematic differences in the comparison 
with the Sun, we have carried out an identical analysis with the solar atlas of Kurucz et al. (1984). The lines were selected (or rejected) and classified following the same criteria for both stars, in an attempt to keep the comparison as meaningful as possible. It is of great interest to analyze the asymmetries of $\mathrm{Fe}$ I lines in the flux spectrum of the Sun on an absolute scale, as this has never been done consistently before and must provide the best standard for comparisons with other stars. Finally, even at risk of repeating what has already been said many times in the literature, we note that there are several good reasons to pick $\mathrm{Fe}$ I among the observed species: the large number of lines in late-type stars, the lack of measurable isotopic shifts and hyperfine splitting, and the availability of accurate laboratory wavelengths.

\subsection{Line Shifts}

We used the line list of Thévenin $(1989,1990)$ to search for $\mathrm{Fe} \mathrm{I}$ lines in the solar spectrum. The central wavelengths and bisectors of the $\mathrm{Fe} \mathrm{I}$ lines in that list were measured in the solar atlas and the McDonald spectrum of Procyon and then compared with the precise laboratory wavelengths measured by Nave et al. (1994). The wavelength calibration of the solar atlas has been examined by Allende Prieto \& García López (1998), who showed that it is highly reliable.

The measurement of the central wavelength of the line profiles was carried out fitting a third-order polynomial to the 11 lowest points around the minimum, equivalent to an interval of 90-120 m $\AA$ in the spectrum of Procyon and 50 $\mathrm{m} \AA$ in the solar spectrum. We measured all the lines in Thévenin's list identified as Fe I lines. For this comparison, the equivalent widths of the lines were estimated by synthesizing the lines with R. L. Kurucz (1992, private communication) model atmospheres corresponding to the Sun and Procyon. This is safer than attempting to measure the equivalent widths in the spectra, as a large fraction of the lines are strongly blended outside their core, especially at the bluer wavelengths. We made use of the solar $g f$-values of Thévenin $(1989,1990)$ and the damping enhancement factors for Fe I recommended by the Blackwell group, as implemented in the LTE synthesis program MOOG (Sneden 1973).

The left-hand panels in Figure 4 show the Fe I line velocity shifts $(v)$ measured in the spectra of the Sun and Procyon. The smaller wavelength coverage available for Procyon (4559-5780 A) in comparison with the solar atlas of Kurucz et al. (1984) (2960-13,000 ̊) reduces the number of measured lines from 1551 to 506 . The line shifts exhibit similar patterns for both stars. There is no reason to think that the well-known granulation observed in the Sun is not present in Procyon, and we naturally associate the larger shifts toward the red for stronger lines with a decreasing of the convective blueshifts. Unfortunately, there is no line in the observed part of Procyon's spectrum stronger than 200 $\mathrm{m} \AA$, leaving open the question of whether the velocity shifts of the strongest Fe I lines fall on a solar-like " plateau." It is interesting to compare the left-hand panels in Figure 4. The velocity shifts in the solar spectrum span a range of $\sim 0.6$ $\mathrm{km} \mathrm{s}^{-1}$, but they span at least $0.9 \mathrm{~km} \mathrm{~s}^{-1}$ for Procyon's spectrum.

The velocity indicated by the strong lines on the solar plateau $\left(W_{\lambda} \geq 200 \mathrm{~m} \AA\right)$ is essentially independent of the line's equivalent width. We note that Figure 4 has been truncated at $W_{\lambda}=250 \mathrm{~m} \AA$, but the solar plateau extends at least to lines as strong as $2 \AA$ (see Allende Prieto \& García López 1998). Part of the observed scatter should be
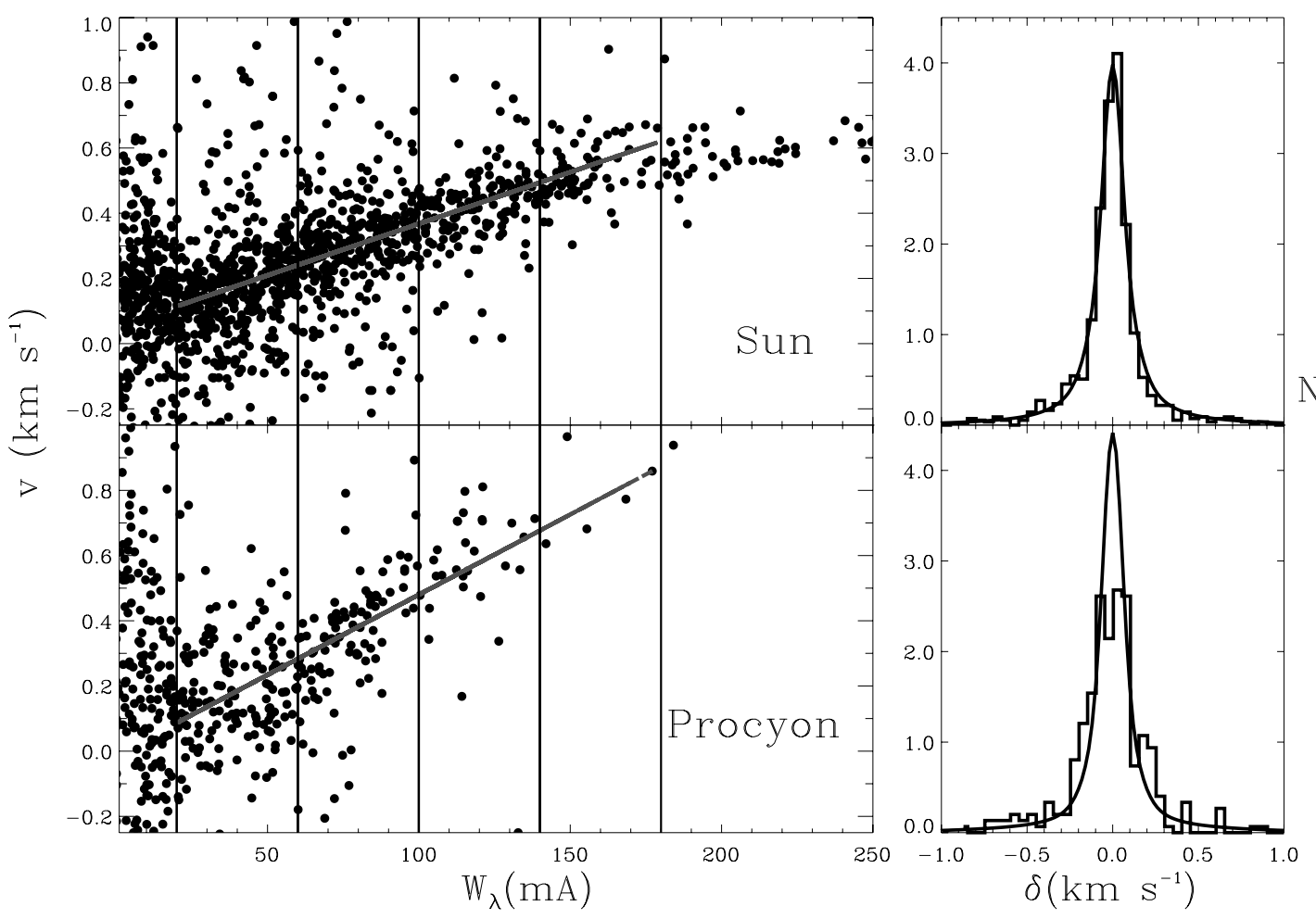

FIG. 4.-Left panels: Velocity shifts between the stellar and laboratory wavelengths for relatively weak lines; for Procyon the zero point is arbitrary. The strong solid line is a least-squares linear fit for all lines with $20 \leq W_{\lambda} \leq 180 \mathrm{~mA}$. Right panels: Difference between the measured shifts and the linear fits to lines with $20 \leq W_{\lambda} \leq 180 \mathrm{~m} \AA$ (histogram); the expected distribution, based on error estimates for the laboratory wavelengths and the polynomial fits to the center of the stellar lines, is also shown (solid curve). 
attributable to the laboratory wavelengths and uncertainties in the measurement of the center of a spectral line (with a finite width), which can be quantified. Nave et al. (1994) classify their wavelengths into four categories, depending on their uncertainty $\left(\sigma_{1}\right)$, which ranges from 0.4 to more than $10 \mathrm{~m} \AA$. Most lines have errors below $5 \mathrm{~m} \AA$, and about half of the lines have errors below $1 \mathrm{~m} \AA$. Errors in the measured stellar wavelengths can be estimated from the horizontal scatter of the polynomial least-squares fit to the line center divided by the square root of the number of points in the fit (in this case 11), $\sigma_{s}$.

Neglecting the effect of unidentified line blends and systematic effects in the wavelength scales of the stellar spectra, the uncertainties in the measured line shifts, $\sigma=$ $\left(\sigma_{s}^{2}+\sigma_{l}^{2}\right)^{1 / 2}$, or more precisely, the distribution of uncertainties, $n(\sigma)$, can be used to predict the distribution of errors $(\delta)$ in the observed velocity shifts as

$$
N(\delta)=\frac{1}{C} \int_{0}^{\infty} \frac{n(\sigma)}{\sigma} \exp \left(-\frac{\delta^{2}}{2 \sigma^{2}}\right) d \sigma,
$$

where $C$ is chosen such that $\int_{-\infty}^{\infty} N(\delta) d \delta=1$. The derived $N(\delta)$ can be compared to the observations in order to constrain the intrinsic scatter.

Discarding a deviant line, the average shift for the 19 measured $\mathrm{Fe} I$ solar lines stronger than $400 \mathrm{~m} \AA$ is $v=0.626 \pm 0.011$ (s.e.m.) $\mathrm{km} \mathrm{s}^{-1}$, consistent with the gravitational redshift of solar photospheric light at Earth, 0.633 $\mathrm{km} \mathrm{s}^{-1}$. The strengthening of the convective blueshifts for weaker lines is definitely not far from linear. A linear regression for lines with equivalent widths $20 \leq W_{\lambda} \leq 180 \mathrm{~m} \AA$ provides, in the solar case, a similar slope as linear fits within several subintervals included in that range. Conversely, there is some marginal indication that the trend flattens for the lines with $20 \leq W_{\lambda} \leq 60 \mathrm{~m} \AA$ in the spectrum of Procyon. The intrinsic scatter for the Sun is very small, at least significantly smaller than our measurement errors (see Fig. 4). For Procyon, there is an additional scatter, but it should be kept in mind that we have neglected other sources of systematic errors, such as those in the theoretical equivalent widths. We will return to this issue in the light of threedimensional model atmospheres.

Making use of the laboratory wavelengths measured recently by S. Johansson (1998, private communication), we derive the velocity shifts for $25 \mathrm{Fe}$ II lines, which exhibit a trend similar to that for $\mathrm{Fe}$ I lines. The velocity shifts of $\mathrm{Fe}$ II lines in the spectrum of the Sun are generally more shifted to the blue than the $\mathrm{Fe} \mathrm{I}$ lines, in agreement with the results obtained by Dravins, Larsson, \& Nordlund (1986) for the center of the disk, but the difference becomes indistinguishable in the spectrum of Procyon.

\subsection{Line Asymmetries}

We have tried to avoid subjective criteria in selecting lines for measuring bisectors. Knowing that average convective line asymmetries for main-sequence $\mathrm{F}-\mathrm{K}$ stars have been neither measured nor predicted to be larger than a few hundred meters per second, we have kept only those lines whose bisectors do not exceed $1 \mathrm{~km} \mathrm{~s}^{-1}$ in amplitude. The very large number of available lines in the spectrum whose rest wavelength is reliably known makes it possible to use such a strong rejection condition. In addition, only lines whose absorption was deeper than $10 \%$ of the continuum level were included in the analysis. These criteria were satisfied by 331 lines in the solar atlas and by 151 in Procyon's

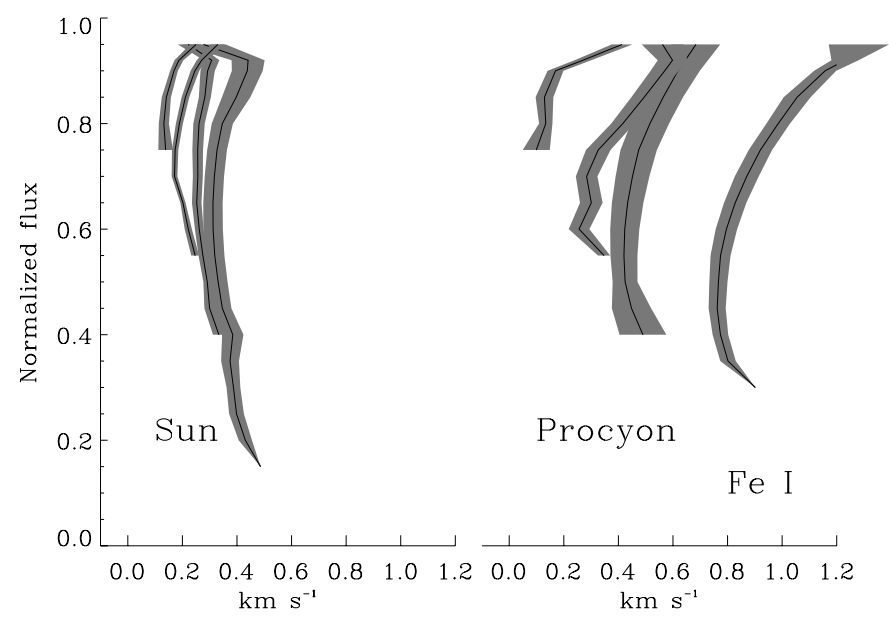

FIG. 5.-Average bisectors for $\mathrm{Fe} \mathrm{I}$ lines with a continuum-normalized flux at the line center $h: h<0.35,0.35<h<0.55,0.55<h<0.75$, and $h>0.75$.

spectra. They were ordered in four different groups depending on their continuum-normalized flux at the line center $h$ : $h<0.35,0.35<h<0.55,0.55<h<0.75$, and $h>0.75$. Their bisectors were averaged retaining the relative velocity differences between the lowest end of the individual bisectors. In the process of averaging we allow for a cleaning, in the sense that at each absorption depth at which the bisectors were measured we reject those deviating by more than $2 \sigma$ from the mean.

Figure 5 shows the averaged bisectors for $\mathrm{Fe}$ I lines. The solar bisectors are similar to the averaged line bisectors measured in the spectrum of the center of the disk by Dravins et al. (1981). The typical "C" shape is clearly visible. A comparison of the velocity spans for the Sun and Procyon is in agreement with previous results: the span of Procyon's lines exceeds the solar values by more than a factor of 2. A similar result holds for the line shifts. As explained above, the velocity differences between the individual bisectors were retained for averaging, but we have not subtracted the gravitational redshift in the solar case, and the zero velocity for Procyon's bisector was arbitrarily set.

The bisectors measured for $\mathrm{Fe}$ II lines are displayed in Figure 6. As they are only a few, we did not average them out. Three solar bisectors (not shown) were rejected by the

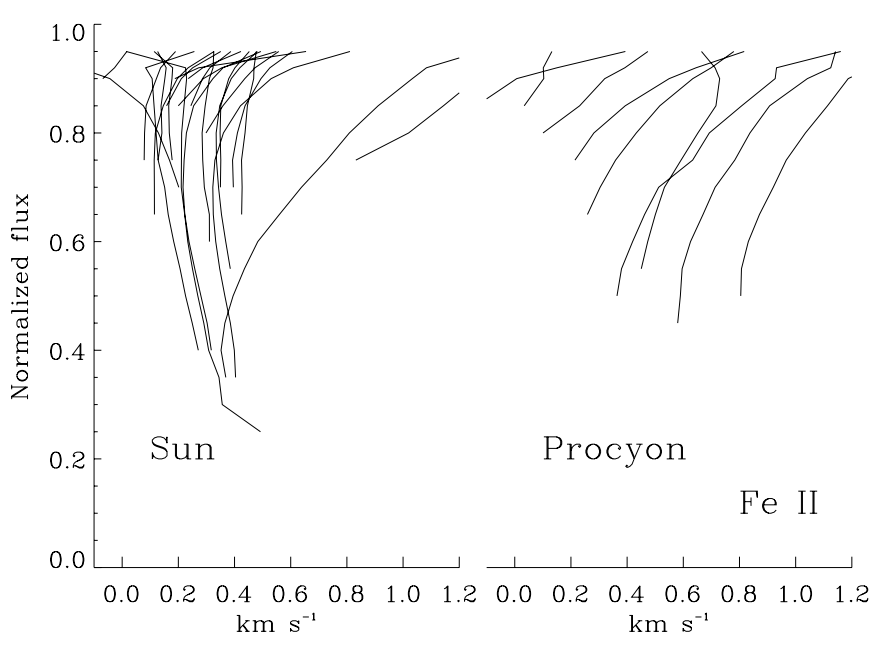

FIG. 6. - Individual bisectors for Fe II lines 
$2 \sigma$ criterion, and there are still two more obvious outliers kept. Fe II bisectors corroborate the qualitative conclusions from $\mathrm{Fe} \mathrm{I}$ lines.

\section{MODELING}

\subsection{Fundamental Stellar Parameters}

Procyon A is in a 40 yr period visual binary system, sharing the systemic velocity with a white dwarf. Girard et al. (2000) have updated its astrometric orbit from photographic plates obtained at different observatories between 1912 and 1995 and made a direct measurement of the angular separation of the pair using the infrared cold coronagraph $(\mathrm{CoCo})$ at the NASA Infrared Telescope Facility and the WFPC 2 on board the Hubble Space Telescope (HST). At a distance of only $3.5 \mathrm{pc}$ from the Sun, the parallax measured by Hipparcos is very precise: $p=(285.93 \pm 0.88) \times 10^{-3}$ arcsec. In addition, Mozurkewich et al. (1991) measured the stellar angular diameter using optical interferometry: $\theta=(5.51 \pm 0.05) \times 10^{-3}$ arcsec. Therefore, its mass can be constrained to

$$
M=\frac{\alpha}{p^{2} P^{2}}\left(1-\frac{\alpha_{A}}{\alpha}\right)=1.42 \pm 0.06 M_{\odot},
$$

where $\alpha, \alpha_{A}$, and $P$ are the semimajor axis of the visual orbit or Procyon A, the semimajor axis of its orbit relative to the barycenter of the system, and the orbital period, respectively, and for which we have adopted $\alpha=4.271 \pm 0$ ".032, $\alpha_{A}=11^{\prime \prime} 232 \pm 0.08$, and $P=40.82 \pm 0.06 \mathrm{yr}$ (Girard et al. 2000), in combination with the Hipparcos parallax. The radius is

$$
R \simeq \frac{\theta}{2 p \tan \left(\theta_{\odot} / 2\right)}=2.071 \pm 0.020 R_{\odot},
$$

where we have adopted $\theta_{\odot} / 2=9599^{\prime \prime} 64 \pm$ 0".02 (Chollet \& Sinceac 1999), leading to a gravity $\log g=3.96 \pm 0.02$ (cgs units). The derived gravity is in good agreement with the estimate of Allende Prieto \& Lambert (1999), $\log g=4.04 \pm 0.14$ dex, obtained from matching the observed $M_{V}$ and $(B-V)$ to the evolutionary models of Bertelli et al. (1994). ${ }^{5}$ Our mass estimate is slightly lower than that given in Girard et al. (2000), as we prefer to adopt the Hipparcos parallax and the angular separation determined from $H S T$-WFPC2 observations, discarding the ground-based measurement. Adopting the parallax derived by Girard et al. (2000), $p=(283.2 \pm 1.5) \times 10^{-3}$ arcsec, we find only a minor correction to the derived mass: $M=1.47 \pm 0.06 M_{\odot}$.

The star has a Johnson visual magnitude of $V=0.363 \pm 0.003 \mathrm{mag}$ (and therefore an absolute magnitude $\left.M_{V}=2.644 \pm 0.007\right)$ and a color index $B-V=$ $0.421 \pm 0.003 \mathrm{mag}$, as derived from 13 measurements extracted from SIMBAD. Fuhrmann et al. (1997) compiled several estimates of Procyon's bolometric flux from the literature. Their weighted average is $F_{\mathrm{BOL}}=(18.20 \pm 0.43)$ $\times 10^{-6} \mathrm{ergs} \mathrm{cm}^{-2} \mathrm{~s}^{-1}$, and therefore the star has an effec-

\footnotetext{
${ }^{5}$ We note, however, that the estimate in a previous paper by Allende Prieto et al. (1999b) for this and other stars in their sample with $T_{\text {eff }}>6000$ $\mathrm{K}$ is underestimated, as a result of the improper use of a different set of isochrones restricted to ages larger than $5 \mathrm{Gyr}$.
}

tive temperature

$$
T_{\text {eff }}=7.400 \times 10^{3}\left(\frac{F_{\mathrm{BOL}}}{\theta^{2}}\right)^{1 / 4}=6512 \pm 49 \mathrm{~K} .
$$

The angular diameter depends on the adopted limbdarkening law. Hence, we revise the derived $T_{\text {eff }}$ and radius in $\S 7$. Finally, a review of the high-resolution spectroscopic analyses previously performed indicates that the star has either solar or slightly lower than solar iron abundance.

Linear interpolation in the solar metallicity isochrones published by Bertelli et al. (1994) to match the pair $\left(M_{V}\right.$, $B-V$ ) constrains very tightly the age of Procyon A to the range $1.66-1.73$ Gyr and predicts a gravity in agreement with the astrometric value. Alternatively, matching the observed radius and mass would shift the age estimate to $2.0 \mathrm{Gyr}$. This last value, less affected by uncertainties in the conversion between the theoretical and observational quantities, is to be preferred. These ages are significantly shorter than the nuclear timescale of $3.9 \mathrm{Gyr}$ adopted by Provencal et al. (1997), implying a mass for the progenitor of the white $\mathrm{dwarf}$ (Procyon B) that is larger than previously estimated.

\subsection{Model Atmospheres}

Realistic ab initio, compressible, radiative hydrodynamical simulations of surface convection in Procyon have been performed with the code formerly used to model solar (e.g., Stein \& Nordlund 1998; Asplund et al. 2000a, 2000b, 2000c; Asplund 2000) and stellar granulation (Asplund et al. 1999; Asplund \& García Pérez 2001). The resulting structure is a three-dimensional, time-dependent model atmosphere with a self-consistent description of the convective flows. The hydrodynamical equations of mass, momentum, and energy conservation,

$$
\begin{gathered}
\frac{\partial \ln \rho}{\partial t}=-\overline{\boldsymbol{v}} \cdot \nabla \ln \rho-\nabla \cdot \overline{\boldsymbol{v}}, \\
\frac{\partial \overline{\boldsymbol{v}}}{\partial t}=-\overline{\boldsymbol{v}} \cdot \nabla \overline{\boldsymbol{v}}+\overline{\boldsymbol{g}}-\frac{P}{\rho} \nabla \ln P+\frac{1}{\rho} \nabla \cdot \sigma, \\
\frac{\partial e}{\partial t}=-\overline{\boldsymbol{v}} \cdot \nabla e-\frac{P}{\rho} \nabla \cdot \overline{\boldsymbol{v}}+Q_{\mathrm{rad}}+Q_{\mathrm{visc}},
\end{gathered}
$$

coupled to the equation of radiative transfer,

$$
(\boldsymbol{n} \cdot \nabla) I_{\lambda}=\eta_{\lambda}-\kappa_{\lambda} I_{\lambda}
$$

have been solved on a nonstaggered Eulerian mesh with $100 \times 100 \times 82$ grid points. In the above equations, $\rho$ denotes the density, $\overline{\boldsymbol{v}}$ the velocity, $\overline{\boldsymbol{g}}$ the gravitational acceleration, $P$ the pressure, $e$ the internal energy, $\sigma$ the viscous stress tensor, $Q_{\mathrm{visc}}$ the viscous dissipation, $Q_{\mathrm{rad}}=\int_{\lambda} \int_{\Omega} \kappa_{\lambda}$ $\left(I_{\lambda}-S_{\lambda}\right) d \Omega d \lambda$ the radiative heating/cooling rate, $I_{\lambda}$ the monochromatic intensity, $\kappa_{\lambda}$ and $\eta \equiv B_{\lambda}(T) \kappa_{\lambda}$ the absorption and emission coefficients, respectively, where $B_{\lambda}(T)$ is the Planck function, and $\boldsymbol{n}$ the unit vector for each of the eight considered directions. The code was stabilized using a hyperviscosity diffusion algorithm (Stein \& Nordlund 1998) with the parameters determined from standard hydrodynamical test cases, like the shock tube. Periodic horizontal boundary conditions and open transmitting top and bottom boundaries were used in an identical fashion to the simulations described by Stein \& Nordlund (1998).

In order to make the atmospheric structure as realistic as possible for direct confrontation with observations, we used 
a detailed equation of state (Mihalas, Dappen, \& Hummer 1988), including the effects of ionization, excitation, and dissociation, as well as continuum (Uppsala opacity package; see Gustafsson et al. 1975; Asplund et al. 1997) and line (R. L. Kurucz 1992, private communication) opacities. The three-dimensional radiative transfer has been solved at each time step of the simulation by Feautrier's method, under the approximations of LTE and the opacity binning technique (Nordlund 1982). The accuracy of the opacity binning procedure has been verified at regular intervals by solving the full monochromatic radiative transfer (2748 wavelength points) in the 1.5-dimensional approximation, i.e., treating each vertical column separately and thus ignoring horizontal radiative transfer effects.

The physical dimension of the numerical box is $21 \times 21 \times 13 \mathrm{Mm}$, of which about $3 \mathrm{Mm}$ correspond to the photosphere. A snapshot from a previous lower resolution simulation sequence of Procyon with a less extended depth scale (Trampedach 1997) was used as initial condition. The full convection simulation covers more than $3 \mathrm{hr}$ of stellar time, but only the final hour has been used for the calculations of spectral line formation presented here. The resulting effective temperature of the $1 \mathrm{hr}$ sequence was $6514 \pm 27 \mathrm{~K}$, in close agreement with the interferometric estimate. We adopted a surface gravity of $\log g=3.96(\mathrm{cgs})$ and solar chemical composition (Grevesse \& Sauval 1998). In particular, the $\mathrm{Fe}$ abundance used for the equation-ofstate and continuum opacity calculations was $\log \epsilon_{\mathrm{Fe}}=$ $7.50{ }^{6}$ The line opacities were extrapolated to the adopted Fe abundance using the standard Kurucz opacity distribution functions (ODFs) with $\log \epsilon_{\mathrm{Fe}}=7.67$ and nonstandard ODFs computed with $\log \epsilon_{\mathrm{Fe}}=7.51$ and with no $\mathrm{He}(\mathrm{R}$. L. Kurucz 1997, private communication; see Trampedach 1997 for details of the procedure).

The resulting convective structures for Procyon are qualitatively similar to the lower resolution runs presented in Nordlund \& Dravins (1990), which, however, were computed with the anelastic approximation and less complete equation of state and opacities. With the new compressible code, larger convective velocities are encountered, and they often exceed the speed of sound, causing prominent shocks in the photosphere. The phenomenon of "naked granulation" discussed by Nordlund \& Dravins (1990) is also present in the improved simulations as seen in Figure 7: the layers with largest temperature contrast due to the convective motions are located around the continuumforming region $\left(\tau_{5000} \simeq 1-3\right)$ and not slightly beneath it, as is the case with cooler stars like the Sun $\left(\tau_{5000} \simeq 10\right)$. In addition, the temperature contrast itself is significantly larger in Procyon than in the Sun. All these features manifest themselves in more pronounced line asymmetries. A detailed description of the convective structures in the granulation simulations of Procyon and other solar-type stars will be presented in a subsequent article, while here we concentrate on the effects upon spectral line formation and the confrontation with observations.

To enable a strictly differential comparison between the three-dimensional and one-dimensional predictions of the resulting line formation, one-dimensional, hydrostatic, plane-parallel model atmospheres have been generated with the MARCS code (Asplund et al. 1997). These homoge-

\footnotetext{
${ }^{6}$ This is on the customary logarithmic abundance scale defined to have $\log \epsilon_{\mathrm{H}}=12.00$.
}

neous models are fully line blanketed and have identical defining parameters as the three-dimensional simulations, including all chemical abundances.

\subsection{Spectral Line Calculations}

Using the granulation snapshots of the Procyon simulation as three-dimensional model atmospheres, the spectral line formation was computed for a sample of $\mathrm{Fe}$ I and $\mathrm{Fe}$ II lines assuming LTE $\left(S_{v}=B_{v}\right)$. The original simulation data were interpolated to a denser vertical grid covering only the outermost $6 \mathrm{Mm}$ but with the same number of depth points as before. From the stored temperatures and densities of the snapshots, monochromatic continuous opacities were computed using the Uppsala opacity package, which was also employed for constructing the one-dimensional model atmospheres with which we compare. The assumptions of Boltzmann and Saha distributions and chemical equilibrium have been made in the calculation of opacities and number densities of the various species.

The radiative transfer was solved for five center-to-limb positions and seven equally spaced azimuthal angles, for a total of 29 different directions. The $\mu$ angles were chosen to facilitate the disk integration taking into account the rotational velocity of the star (Dravins \& Nordlund 1990).

The flux profiles at different velocities were computed as

$$
F(v)=\int_{\varphi=0}^{2 \pi} \int_{\mu=0}^{1} I\left(v-v_{\text {rot }} \sin i \sin \theta \cos \varphi, \mu, \varphi\right) \mu d \mu d \varphi,
$$

with $\mu=\cos \theta$. The integrals were evaluated using a fourpoint Gaussian quadrature for $\mu$ and seven equidistant $\varphi$ angles with equal weight. Finally, the rotationally broadened profiles for the 31 individual snapshots were added together before normalization to the local continuum. It was verified through test calculations that using twice as many $\mu$ and $\varphi$ angles results in insignificant differences in the theoretical line asymmetries. The procedure accounts for the Doppler shifts introduced by the convective motions in the stellar atmosphere which, together with the temperature inhomogeneities, produce the characteristic line bisector shapes. Various tests showed that the temporal coverage of the convection simulation produces statistically meaningful line profiles and asymmetries. Following the calculations of disk integration and rotational broadening, the resulting profiles were convolved with a Gaussian with an FWHM of $1.5 \mathrm{~km} \mathrm{~s}^{-1}$, to account for the finite spectral resolution of the spectrograph. As shown for the Sun, for which the rotational velocity is accurately known, there is no need to introduce any additional line broadening in the form of micro- and macroturbulence in analyses based on high-resolution three-dimensional model atmospheres (Asplund et al. 2000a, 2000b, 2000c; Asplund 2000).

The profiles were computed for 141 equidistant velocities, covering from -28 to $28 \mathrm{~km} \mathrm{~s}^{-1}$ around the laboratory wavelengths. Each line was calculated for three different $\mathrm{Fe}$ abundances, 7.20, 7.50, and 7.80, from which the final profile was interpolated. Test calculations verified that the abundance interval was sufficiently small not to introduce any systematic errors in the abundances (less than $0.01 \mathrm{dex}$ ) and line asymmetries (less than $0.02 \mathrm{~km} \mathrm{~s}^{-1}$ ) derived.

The one-dimensional spectral line calculations for the comparison with the three-dimensional results have been 

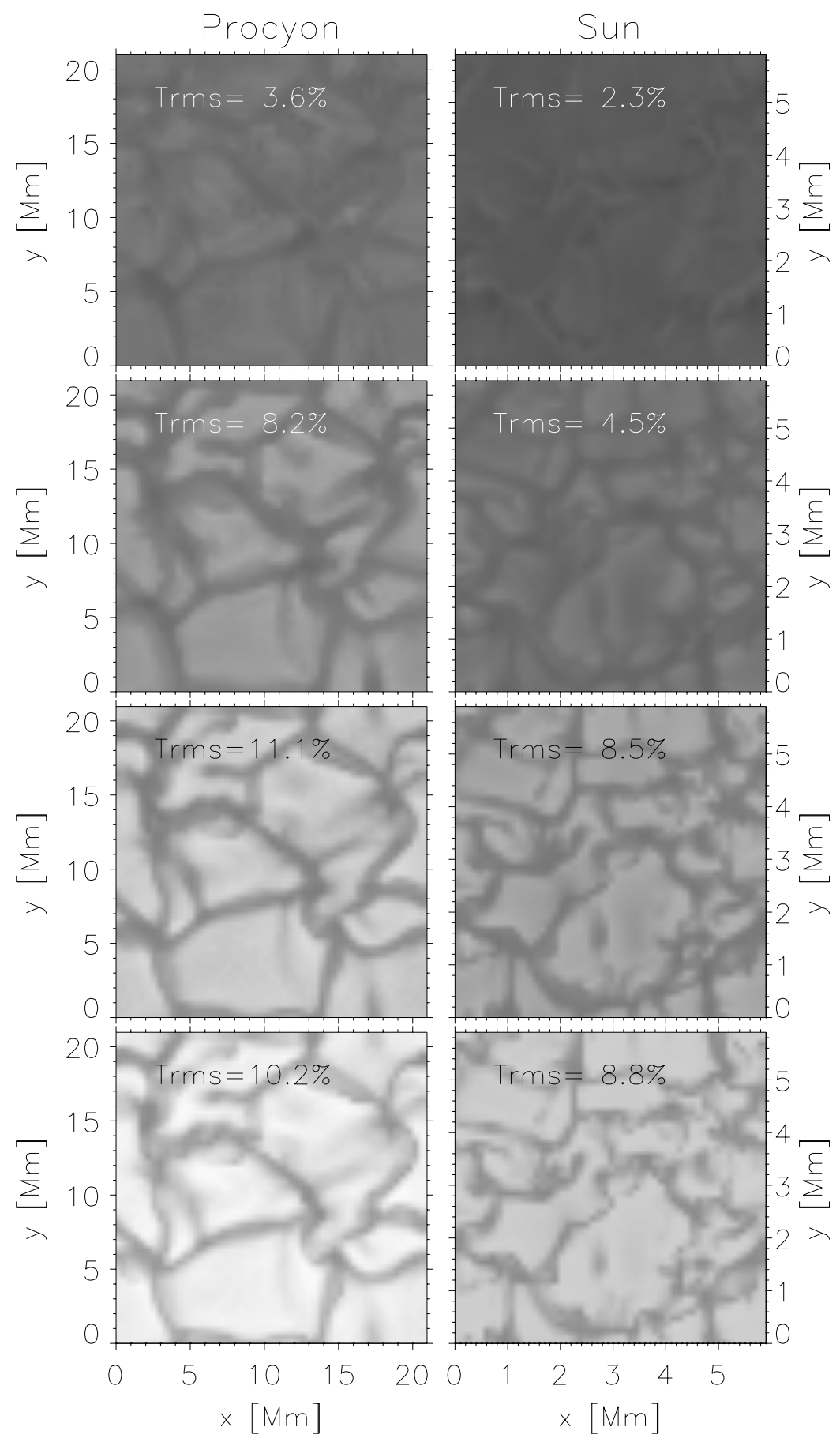

FIG. 7.-Temperature on surfaces of equal optical depths in the convection simulations of Procyon (left panels) and the Sun (right panels). The surfaces correspond to continuum optical depths at $5000 \AA$ of $0.3,1,3$, and 10 from top to bottom. It should be noted that these iso-tau surfaces are highly corrugated and therefore the temperature contrast is much greater across surfaces of equal geometrical depths. All images share the same maximum and minimum temperature cuts, which highlights the significantly larger temperature contrast in Procyon. For each iso-tau surface, the temperature contrast $\left(T_{\mathrm{rms}} /\langle T\rangle\right)$ is given.

computed with the same spectral synthesis code as the three-dimensional profiles. Without the convective Doppler shifts, additional ad hoc broadening in the form of the micro- and macroturbulence must be invoked in one dimension in order to obtain correct line widths, with the former affecting the line strengths and the latter affecting only the line shapes. In both cases, Gaussian distributions are assumed.

\subsection{Line Data}

Iron is the best represented element in the spectrum of late-type stars. Neutral iron has been the subject of a number of fine laboratory works to derive radiative transition probabilities at Oxford (e.g., Blackwell et al. 1986). The number of lines measured with high accuracy and in a homogeneous manner has been enlarged by the work of 
TABLE 1

Spectral Lines Selected for the Iron Abundance Analysis of Procyon

\begin{tabular}{|c|c|c|c|c|c|c|}
\hline Species & 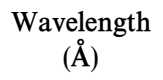 & $\begin{array}{l}\text { Excitation Potential } \\
(\mathrm{eV})\end{array}$ & $\begin{array}{c}\log g f \\
(\mathrm{dex})\end{array}$ & $\begin{array}{c}\log \epsilon 3 \mathrm{D} \\
(\mathrm{dex})\end{array}$ & $\begin{array}{l}\log \epsilon \in 1 \mathrm{D} \\
(\mathrm{dex})\end{array}$ & $\begin{array}{c}W_{\lambda}(3 \mathrm{D}) \\
(\mathrm{m} \AA)\end{array}$ \\
\hline $\mathrm{Fe}_{\mathrm{I}} \ldots \ldots$ & 4602.00 & 1.608 & -3.13 & 7.30 & 7.31 & 47 \\
\hline $\mathrm{Fe}_{\mathrm{I}} \ldots \ldots$ & 4602.94 & 1.485 & -2.21 & 7.51 & 7.27 & 105 \\
\hline $\mathrm{Fe}_{\mathrm{I}} \ldots \ldots$ & 4635.85 & 2.845 & -2.36 & 7.32 & 7.34 & 31 \\
\hline $\mathrm{Fe}_{\mathrm{I}} \ldots \ldots$ & 4683.56 & 2.831 & -2.32 & 7.24 & 7.25 & 30 \\
\hline $\mathrm{Fe}_{\mathrm{I}} \ldots \ldots$ & 4690.14 & 3.686 & -1.64 & 7.49 & 7.44 & 39 \\
\hline $\mathrm{Fe}_{\mathrm{I}} \ldots \ldots$ & 4710.28 & 3.018 & -1.61 & 7.52 & 7.41 & 72 \\
\hline $\mathrm{Fe}_{\mathrm{I}} \ldots \ldots$ & 4735.84 & 4.076 & -1.33 & 7.65 & 7.57 & 46 \\
\hline $\mathrm{Fe}_{\mathrm{I}} \ldots \ldots$ & 4741.53 & 2.831 & -1.76 & 7.16 & 7.11 & 54 \\
\hline $\mathrm{Fe}_{\mathrm{I}} \ldots \ldots$ & 4745.80 & 3.654 & -1.27 & 7.47 & 7.39 & 58 \\
\hline $\mathrm{Fe}_{\mathrm{I}} \ldots \ldots$ & 4779.44 & 3.415 & -2.02 & 7.22 & 7.23 & 21 \\
\hline $\mathrm{Fe}_{\mathrm{I}} \ldots \ldots$ & 4786.81 & 3.017 & -1.61 & 7.50 & 7.39 & 70 \\
\hline $\mathrm{Fe}_{\mathrm{I}} \ldots \ldots$ & 4788.76 & 3.237 & -1.76 & 7.39 & 7.35 & 48 \\
\hline $\mathrm{Fe}_{\mathrm{I}} \ldots \ldots$ & 4789.65 & 3.546 & -0.96 & 7.49 & 7.31 & 77 \\
\hline $\mathrm{Fe}_{\mathrm{I}} \ldots \ldots$ & 4798.27 & 4.186 & -1.17 & 7.16 & 7.12 & 25 \\
\hline $\mathrm{Fe}_{\mathrm{I}} \ldots \ldots$ & 4802.88 & 3.642 & -1.51 & 7.40 & 7.36 & 43 \\
\hline Fe I ....... & 4832.73 & 3.640 & -1.73 & 7.61 & 7.57 & 42 \\
\hline $\mathrm{Fe}_{\mathrm{I}} \ldots \ldots$ & 4844.01 & 3.546 & -2.05 & 7.46 & 7.45 & 25 \\
\hline $\mathrm{Fe}_{\mathrm{I}} \ldots \ldots$ & 4872.14 & 2.882 & -0.57 & 7.45 & 7.22 & 133 \\
\hline $\mathrm{Fe}_{\mathrm{I}} \ldots \ldots$ & 4903.31 & 2.882 & -0.93 & 7.64 & 7.40 & 120 \\
\hline $\mathrm{Fe}_{\mathrm{I}} \ldots \ldots$ & 4918.99 & 2.865 & -0.34 & 7.52 & 7.33 & 160 \\
\hline $\mathrm{Fe}_{\mathrm{I}} \ldots \ldots$ & 4985.25 & 3.928 & -0.56 & 7.49 & 7.27 & 85 \\
\hline $\mathrm{Fe}_{\mathrm{I}} \ldots \ldots$ & 4994.13 & 0.915 & -2.97 & 7.26 & 7.15 & 87 \\
\hline $\mathrm{Fe}_{\mathrm{I}} \ldots \ldots$ & 5006.12 & 2.832 & -0.62 & 7.61 & 7.48 & 143 \\
\hline $\mathrm{Fe}_{\mathrm{I}} \ldots \ldots$ & 5014.94 & 3.943 & -0.30 & 7.48 & 7.26 & 98 \\
\hline $\mathrm{Fe}_{\mathrm{I}} \ldots \ldots$ & 5022.79 & 2.990 & -2.20 & 7.46 & 7.44 & 41 \\
\hline $\mathrm{Fe}_{\mathrm{I}} \ldots \ldots$ & 5049.82 & 2.279 & -1.36 & 7.59 & 7.30 & 118 \\
\hline $\mathrm{Fe}_{\mathrm{I}} \ldots \ldots$ & 5054.64 & 3.640 & -1.92 & 7.20 & 7.21 & 17 \\
\hline $\mathrm{Fe}_{\mathrm{I}} \ldots \ldots$ & 5079.74 & 0.990 & -3.24 & 7.42 & 7.31 & 78 \\
\hline $\mathrm{Fe}_{\mathrm{I}} \ldots \ldots$ & 5083.34 & 0.958 & -2.84 & 7.25 & 7.12 & 91 \\
\hline Fe I .......... & 5127.36 & 0.915 & -3.25 & 7.28 & 7.23 & 75 \\
\hline $\mathrm{Fe}_{\mathrm{I}} \ldots \ldots$ & 5141.74 & 2.424 & -2.24 & 7.41 & 7.34 & 62 \\
\hline $\mathrm{Fe}_{\mathrm{I}} \ldots \ldots$ & 5145.09 & 2.198 & -2.88 & 7.05 & 7.08 & 22 \\
\hline Fe I ....... & 5151.91 & 1.011 & -3.32 & 7.35 & 7.30 & 71 \\
\hline $\mathrm{Fe}_{\mathrm{I}} \ldots \ldots$ & 5194.94 & 1.557 & -2.02 & 7.36 & 7.14 & 109 \\
\hline $\mathrm{Fe}_{\mathrm{I}} \ldots \ldots$ & 5198.71 & 2.223 & -2.09 & 7.35 & 7.24 & 77 \\
\hline $\mathrm{Fe}_{\mathrm{I}} \ldots . .$. & 5232.94 & 2.940 & -0.06 & 7.41 & 7.25 & 175 \\
\hline Fe I ...... & 5242.49 & 3.634 & -0.97 & 7.46 & 7.31 & 75 \\
\hline $\mathrm{Fe}_{\mathrm{I}} \ldots \ldots$ & 5288.52 & 3.694 & -1.51 & 7.31 & 7.29 & 37 \\
\hline $\mathrm{Fe}_{\mathrm{I}} \ldots \ldots$ & 5321.11 & 4.434 & -1.09 & 7.22 & 7.20 & 23 \\
\hline $\mathrm{Fe}_{\mathrm{I}} \ldots \ldots$ & 5322.04 & 2.279 & -2.80 & 7.22 & 7.26 & 31 \\
\hline Fe I ...... & 5328.04 & 0.915 & -1.47 & 7.50 & 7.29 & 184 \\
\hline $\mathrm{Fe}_{\mathrm{I}} \ldots \ldots$ & 5364.87 & 4.445 & 0.23 & 7.51 & 7.31 & 110 \\
\hline $\mathrm{Fe}_{\mathrm{I}} \ldots \ldots$ & 5365.40 & 3.573 & -1.02 & 7.21 & 7.11 & 63 \\
\hline Fe I ........ & 5367.47 & 4.415 & 0.44 & 7.43 & 7.22 & 121 \\
\hline $\mathrm{Fe}_{\mathrm{I}} \ldots \ldots$ & 5383.37 & 4.312 & 0.64 & 7.47 & 7.24 & 139 \\
\hline $\mathrm{Fe}_{\mathrm{I}} \ldots \ldots$ & 5397.13 & 0.915 & -1.98 & 7.60 & 7.29 & 152 \\
\hline $\mathrm{Fe}_{\mathrm{I}} \ldots \ldots$ & 5403.82 & 4.076 & -1.03 & 7.49 & 7.38 & 54 \\
\hline Fe I ....... & 5415.20 & 4.386 & 0.64 & 7.40 & 7.21 & 138 \\
\hline Fe I ....... & 5434.52 & 1.011 & -2.13 & 7.59 & 7.29 & 139 \\
\hline Fe I ...... & 5446.92 & 0.990 & -1.91 & 7.64 & 7.33 & 157 \\
\hline Fe I ...... & 5464.28 & 4.143 & -1.40 & 7.21 & 7.21 & 21 \\
\hline Fe I ..... & 5466.99 & 3.573 & -2.23 & 7.55 & 7.58 & 21 \\
\hline $\mathrm{Fe}_{\mathrm{I}} \ldots \ldots$ & 5483.10 & 4.154 & -1.41 & 7.37 & 7.35 & 27 \\
\hline $\mathrm{Fe}_{\mathrm{I}} \ldots \ldots$ & 5497.52 & 1.011 & -2.83 & 7.55 & 7.45 & 105 \\
\hline Fe I ...... & 5501.47 & 0.958 & -3.05 & 7.48 & 7.33 & 94 \\
\hline $\mathrm{Fe}_{\mathrm{I}} \ldots \ldots$ & 5586.76 & 3.368 & -0.14 & 7.53 & 7.30 & 144 \\
\hline $\mathrm{Fe}_{\mathrm{I}} \ldots \ldots$ & 5618.63 & 4.209 & -1.27 & 7.38 & 7.36 & 31 \\
\hline $\mathrm{Fe}_{\mathrm{I}} \ldots \ldots$ & 5701.54 & 2.559 & -2.14 & 7.34 & 7.29 & 59 \\
\hline $\mathrm{Fe}_{\mathrm{I}} \ldots \ldots$ & 5741.85 & 4.256 & -1.67 & 7.41 & 7.41 & 16 \\
\hline Fe II...... & 4576.33 & 2.844 & -2.91 & 7.52 & 7.26 & 98 \\
\hline $\mathrm{Fe}$ II ....... & 4620.51 & 2.828 & -3.19 & 7.42 & 7.25 & 80 \\
\hline Fe II....... & 4629.34 & 2.807 & -2.28 & 7.54 & 7.24 & 133 \\
\hline Fe II....... & 4923.92 & 2.891 & -1.26 & 7.50 & 7.41 & 234 \\
\hline Fe II...... & 5234.62 & 3.221 & -2.23 & 7.62 & 7.36 & 127 \\
\hline Fe II..... & 5264.80 & 3.230 & -3.23 & 7.61 & 7.46 & 71 \\
\hline $\mathrm{Fe}$ II ...... & 5414.07 & 3.221 & -3.48 & 7.37 & 7.28 & 45 \\
\hline $\mathrm{Fe}$ II ....... & 5525.12 & 3.267 & -3.94 & 7.35 & 7.33 & 22 \\
\hline
\end{tabular}


TABLE 2

Spectral Lines Selected for the Iron Abundance ANalysis of the SuN

\begin{tabular}{|c|c|c|c|c|c|c|}
\hline Species & $\begin{array}{l}\text { Wavelength } \\
\text { (§) }\end{array}$ & $\begin{array}{c}\text { Excitation Potential } \\
(\mathrm{eV})\end{array}$ & $\begin{array}{c}\log g f \\
(\mathrm{dex})\end{array}$ & $\begin{array}{c}\log \epsilon 3 \mathrm{D} \\
(\mathrm{dex})\end{array}$ & $\begin{array}{c}\log \epsilon 1 \mathrm{D} \\
(\mathrm{dex})\end{array}$ & $\begin{array}{c}W_{\lambda}(3 \mathrm{D}) \\
(\mathrm{m} \AA)\end{array}$ \\
\hline $\mathrm{Fe}_{\mathrm{I}} \ldots \ldots$ & 4389.25 & 0.052 & -4.55 & 7.36 & 7.45 & 75 \\
\hline Fe I ....... & 4445.47 & 0.087 & -5.38 & 7.29 & 7.41 & 41 \\
\hline Fe I ...... & 5044.21 & 2.851 & -2.02 & 7.37 & 7.40 & 75 \\
\hline Fe I ...... & 5247.05 & 0.087 & -4.98 & 7.43 & 7.54 & 68 \\
\hline $\mathrm{Fe}_{\mathrm{I}} \ldots \ldots$ & 5250.21 & 0.121 & -4.90 & 7.35 & 7.47 & 67 \\
\hline $\mathrm{Fe}_{\mathrm{I}} \ldots \ldots$ & 5253.46 & 3.283 & -1.57 & 7.38 & 7.40 & 79 \\
\hline $\mathrm{Fe}_{\mathrm{I}} \ldots . .$. & 5329.99 & 4.076 & -1.22 & 7.44 & 7.47 & 58 \\
\hline Fe I ...... & 5412.79 & 4.434 & -1.72 & 7.34 & 7.36 & 18 \\
\hline $\mathrm{Fe}_{\mathrm{I}} \ldots \ldots$ & 5491.83 & 4.186 & -2.19 & 7.41 & 7.43 & 13 \\
\hline $\mathrm{Fe}_{\mathrm{I}} \ldots . .$. & 5525.54 & 4.230 & -1.08 & 7.32 & 7.35 & 53 \\
\hline Fe I ...... & 5661.35 & 4.284 & -1.76 & 7.37 & 7.39 & 23 \\
\hline $\mathrm{Fe}_{\mathrm{I}} \ldots \ldots$ & 5701.54 & 2.559 & -2.14 & 7.48 & 7.52 & 87 \\
\hline $\mathrm{Fe}_{\mathrm{I}} \ldots \ldots$ & 5705.46 & 4.301 & -1.36 & 7.35 & 7.38 & 38 \\
\hline $\mathrm{Fe}_{\mathrm{I}} \ldots . .$. & 5778.45 & 2.588 & -3.44 & 7.35 & 7.43 & 22 \\
\hline Fe I ...... & 5784.66 & 3.396 & -2.53 & 7.36 & 7.41 & 27 \\
\hline Fe I ....... & 5855.08 & 4.607 & -1.48 & 7.39 & 7.41 & 23 \\
\hline $\mathrm{Fe}_{\mathrm{I}} \ldots . .$. & 5956.69 & 0.859 & -4.50 & 7.33 & 7.47 & 54 \\
\hline Fe I ...... & 6082.71 & 2.223 & -3.55 & 7.37 & 7.47 & 35 \\
\hline $\mathrm{Fe}_{\mathrm{I}} \ldots \ldots$ & 6136.99 & 2.198 & -2.93 & 7.37 & 7.45 & 65 \\
\hline $\mathrm{Fe}_{\mathrm{I}} \ldots \ldots$ & 6151.62 & 2.176 & -3.37 & 7.47 & 7.58 & 51 \\
\hline $\mathrm{Fe}_{\mathrm{I}} \ldots . .$. & 6173.34 & 2.223 & -2.88 & 7.43 & 7.52 & 69 \\
\hline $\mathrm{Fe}_{\mathrm{I}} \ldots \ldots$ & 6200.31 & 2.608 & -2.37 & 7.42 & 7.51 & 74 \\
\hline $\mathrm{Fe}_{\mathrm{I}} \ldots \ldots$ & 6219.28 & 2.198 & -2.45 & 7.47 & 7.50 & 91 \\
\hline $\mathrm{Fe}_{\mathrm{I}} \ldots \ldots$ & 6240.65 & 2.223 & -3.17 & 7.28 & 7.38 & 49 \\
\hline Fe I ...... & 6265.13 & 2.176 & -2.54 & 7.46 & 7.53 & 88 \\
\hline Fe I ...... & 6271.28 & 3.332 & -2.70 & 7.37 & 7.42 & 24 \\
\hline $\mathrm{Fe}_{\mathrm{I}} \ldots \ldots$ & 6280.62 & 0.859 & -4.39 & 7.43 & 7.51 & 65 \\
\hline $\mathrm{Fe}_{\mathrm{I}} \ldots . .$. & 6297.79 & 2.223 & -2.64 & 7.32 & 7.41 & 75 \\
\hline Fe I ...... & 6322.69 & 2.588 & -2.47 & 7.56 & 7.64 & 77 \\
\hline Fe I .......... & 6481.87 & 2.279 & -3.01 & 7.47 & 7.55 & 64 \\
\hline $\mathrm{Fe}_{\mathrm{I}} \ldots . .$. & 6498.94 & 0.958 & -4.69 & 7.41 & 7.56 & 46 \\
\hline Fe I ...... & 6574.23 & 0.990 & -5.02 & 7.39 & 7.54 & 28 \\
\hline Fe I ....... & 6581.21 & 1.485 & -4.68 & 7.28 & 7.39 & 18 \\
\hline $\mathrm{Fe}_{\mathrm{I}} \ldots \ldots$ & 6593.87 & 2.433 & -2.37 & 7.48 & 7.54 & 87 \\
\hline $\mathrm{Fe}_{\mathrm{I}} \ldots . .$. & 6609.11 & 2.559 & -2.66 & 7.45 & 7.53 & 67 \\
\hline $\mathrm{Fe}_{\mathrm{I}} \ldots \ldots$ & 6625.02 & 1.011 & -5.34 & 7.36 & 7.51 & 15 \\
\hline $\mathrm{Fe}_{\mathrm{I}} \ldots \ldots$ & 6667.71 & 4.584 & -2.11 & 7.49 & 7.51 & 9 \\
\hline $\mathrm{Fe}_{\mathrm{I}} \ldots \ldots$ & 6699.14 & 4.593 & -2.10 & 7.44 & 7.46 & 8 \\
\hline Fe I ...... & 6739.52 & 1.557 & -4.79 & 7.25 & 7.35 & 12 \\
\hline Fe I ...... & 6750.15 & 2.424 & -2.59 & 7.44 & 7.54 & 76 \\
\hline Fe I ...... & 6793.26 & 4.076 & -2.33 & 7.37 & 7.40 & 13 \\
\hline $\mathrm{Fe}_{\mathrm{I}} \ldots . .$. & 6804.27 & 4.584 & -1.81 & 7.40 & 7.42 & 14 \\
\hline $\mathrm{Fe}_{\mathrm{I}} \ldots . .$. & 6837.01 & 4.593 & -1.69 & 7.39 & 7.42 & 17 \\
\hline $\mathrm{Fe}_{\mathrm{I}} \ldots \ldots$ & 6854.82 & 4.593 & -1.93 & 7.48 & 7.50 & 13 \\
\hline $\mathrm{Fe}_{\mathrm{I}} \ldots . .$. & 6945.21 & 2.424 & -2.45 & 7.43 & 7.51 & 83 \\
\hline $\mathrm{Fe}_{\mathrm{I}} \ldots \ldots$ & 6971.93 & 3.018 & -3.34 & 7.33 & 7.40 & 13 \\
\hline Fe I ...... & 6978.85 & 2.484 & -2.45 & 7.45 & 7.49 & 80 \\
\hline $\mathrm{Fe}_{\mathrm{I}} \ldots \ldots$ & 7189.15 & 3.071 & -2.77 & 7.48 & 7.54 & 40 \\
\hline $\mathrm{Fe}_{\mathrm{I}} \ldots \ldots$ & 7401.69 & 4.186 & -1.60 & 7.47 & 7.51 & 42 \\
\hline Fe I ...... & 7723.21 & 2.279 & -3.62 & 7.51 & 7.62 & 41 \\
\hline Fe II...... & 4576.33 & 2.844 & -2.91 & 7.40 & 7.35 & 66 \\
\hline $\mathrm{Fe}$ II ....... & 4620.51 & 2.828 & -3.19 & 7.30 & 7.28 & 52 \\
\hline $\mathrm{Fe}$ II ....... & 4656.98 & 2.891 & -3.58 & 7.32 & 7.29 & 34 \\
\hline $\mathrm{Fe}$ II...... & 5234.62 & 3.221 & -2.23 & 7.50 & 7.46 & 86 \\
\hline Fe II...... & 5264.80 & 3.230 & -3.23 & 7.58 & 7.56 & 47 \\
\hline Fe II...... & 5414.07 & 3.221 & -3.48 & 7.35 & 7.33 & 28 \\
\hline $\mathrm{Fe}$ II...... & 5525.12 & 3.267 & -3.94 & 7.34 & 7.33 & 12 \\
\hline Fe II...... & 5627.49 & 3.387 & -4.10 & 7.44 & 7.42 & 9 \\
\hline $\mathrm{Fe}$ II....... & 6432.68 & 2.891 & -3.51 & 7.39 & 7.37 & 41 \\
\hline $\mathrm{Fe}$ II....... & 6516.08 & 2.891 & -3.38 & 7.54 & 7.51 & 54 \\
\hline Fe II...... & 7222.39 & 3.889 & -3.37 & 7.63 & 7.62 & 20 \\
\hline $\mathrm{Fe}$ II....... & 7224.48 & 3.889 & -3.29 & 7.53 & 7.52 & 20 \\
\hline Fe II...... & 7449.33 & 3.889 & -3.07 & 7.25 & 7.23 & 18 \\
\hline $\mathrm{Fe}$ II...... & 7515.83 & 3.903 & -3.45 & 7.47 & 7.46 & 13 \\
\hline $\mathrm{Fe}$ II....... & 7711.72 & 3.903 & -2.45 & 7.38 & 7.35 & 47 \\
\hline
\end{tabular}


O'Brian et al. (1991). Shown to be in good agreement with the Oxford scale (see Lambert et al. 1996), we have adopted this last reference. Unfortunately, the situation for ionized iron is worse. To select transition probabilities for this species, we have restricted the search to laboratory measurements. Theoretical calculations still show many systematic effects as well as a large scatter. Astrophysical determinations always imply a certain prejudice that we wish to avoid: a selection of a model atmosphere, abundances, collisional damping parameters, etc. As we felt that an updated critical compilation of laboratory $\mathrm{Fe}$ II $f$-values was lacking, we have looked for data for all lines with wavelengths longer than $300 \mathrm{~nm}$, rather than restricting the search to the spectral range of Procyon's observations. The method adopted to average the Fe II transition probabilities and the data are described in the Appendix. By combining laboratory, astrophysical, and theoretical determinations, it is possible to amass a larger line list, but at the expense of higher uncertainties (see, e.g., Giridhar \& Ferro 1995).

Whenever possible, new quantum mechanical calculations (Anstee \& O'Mara 1991; Barklem \& O'Mara 1997; Barklem, O'Mara, \& Ross 1998; Barklem, Piskunov, \& O'Mara 2000) have been employed for the pressure broadening of the spectral lines by collisions with neutral hydrogen. Otherwise (in particular for the $\mathrm{Fe}$ II lines as the quantum mechanical treatment has not yet been generalized to ionized species), the classical formula proposed by Unsöld (1955), with an additional enhancement factor $E=2.0$, has been adopted. Radiative damping was included with values obtained from the Vienna Atomic Line Database (VALD; Kupka et al. 1999). Stark broadening was not considered.

We selected lines of neutral and singly ionized iron from the observed spectrum that were included in the list of O'Brian et al. (1991) (Fe I) or in our list in the Appendix ( $\mathrm{Fe}$ II). Many lines were eliminated from a preliminary list, since they were apparently blended with other features. Visual inspection decided which lines and parts of the profiles were selected at this point. The lines are listed in Table 1.

In addition, we extracted the profiles of several $\mathrm{Fe} \mathrm{I}$ and Fe II lines from the solar atlas of Kurucz et al. (1984), in order to estimate the solar iron abundance in a similar fashion. The lines were selected from the list in Asplund et al. (2000c). They show clean profiles, and the sources for the atomic data are identical to those used for Procyon. These lines and their atomic data appear in Table 2. In some cases, the transition probabilities differ from those given in Asplund et al. (2000c); the difference is, however, marginal ( $\simeq 0.02$ dex on average for both $\mathrm{Fe} \mathrm{I}$ and $\mathrm{Fe}$ II). We noticed some solar Fe lines partially affected by blends, and the afflicted parts of the profiles were ignored in the analysis.

\section{COMPARISON BETWEEN THEORETICAL AND OBSERVED LINE PROFILES}

\subsection{Line Profiles and Stellar Rotation}

In the case of three-dimensional analyses, $v_{\text {rot }} \sin i$ can be determined by minimizing the residuals between theoretical and observed profiles, since no other unknown broadening like macro- and microturbulence enters the calculations. This is performed through a $\chi^{2}$ analysis with the elemental abundance, $v_{\text {rot }} \sin i$, FWHM, and $v_{\text {rad }}$ as free parameters.
By FWHM we refer to that of an assumed-Gaussian ${ }^{7}$ profile that in the simulations accounts for the instrumental profile, while $v_{\text {rad }}$ is the required overall velocity shift of the whole observed profile. In the similar one-dimensional analysis described below the FWHM represents the combined effect of instrumental resolution and macroturbulence, while the microturbulence is kept constant in the $\chi^{2}$ analysis. Figures 8 and 9 compare the observations and the best-fit synthetic spectra calculated with onedimensional and three-dimensional model atmospheres, respectively, for several iron lines. A common deficiency of the one-dimensional predictions is apparent from Figure 8, where the residuals show an oscillation around to the line center. Figure 10 shows the best-fit parameters obtained from individual lines.

For Procyon, we estimate $v_{\text {rot }} \sin i=3.16 \pm 0.50 \mathrm{~km} \mathrm{~s}^{-1}$, where the quoted uncertainty is the standard deviation for the clean samples of Fe I and Fe II lines minus a few obviously discrepant cases (see Fig. 10). There is, except for a couple of aberrant lines, no need for additional Gaussian broadening above the known instrumental broadening (1.5 $\mathrm{km} \mathrm{s}^{-1}$ ); macroturbulence, which must be introduced with the one-dimensional model, is not required. The residual fluxes show no systematic behavior across the profile, as the corresponding one-dimensional lines do. In fact, the threedimensional modeling makes it possible to detect blended lines which would have escaped detection in onedimensional analyses.

It should be noted, however, that the above estimate of $v_{\text {rot }} \sin i$ may be slightly overestimated as a result of the finite numerical resolution of the convection simulation. With higher resolution more of the high-velocity tails of the velocity distributions are sampled, which causes additional broadening of the lines (Asplund et al. 2000a). For the Sun, the predicted line profiles appear to have converged at the currently highest affordable resolution $(200 \times 200 \times 82)$. Since the present Procyon simulations are based on a grid size of $100 \times 100 \times 82$ as a result of computing time considerations, the widths of the theoretical line profiles may have been slightly underestimated. Such a conclusion is supported by the presence of a persistent trend in derived Fe abundances with line strength ( $(6)$. Assuming a similar behavior with numerical resolution for Procyon as for the Sun, $v_{\text {rot }} \sin i$ may be $\approx 0.5 \mathrm{~km} \mathrm{~s}^{-1}$ less than estimated above. However, this additional broadening has a very limited impact on the predicted line shifts and asymmetries (Asplund et al. 2000a), in particular for weak lines, which are still reliable indicators of the chemical abundances.

A detailed Fourier analysis by Gray (1981a), using classical one-dimensional model atmospheres and McDonald spectra with a resolution of $2.5 \mathrm{~km} \mathrm{~s}^{-1}$, provided $v_{\text {rot }} \sin i=2.8 \pm 0.3 \mathrm{~km} \mathrm{~s}^{-1}$ and a radial tangential macroturbulence of $7.0 \pm 0.1 \mathrm{~km} \mathrm{~s}^{-1}$. Studies by Fekel (1997) and Benz \& Mayor (1984) gave estimates for $v_{\text {rot }} \sin i$ of $4.9 \pm 1.0$ and $4.5 \pm 1.1 \mathrm{~km} \mathrm{~s}^{-1}$, respectively. Our own $\chi^{2}$ analysis using one-dimensional model atmospheres reveals similar values but also that the derived $v_{\text {rot }} \sin i$ depends on the choice of line, in particular for weak lines. At least partly, this will depend on the form of the adopted macroturbulence, for example, Gaussian as here, or radial tangen-

\footnotetext{
${ }^{7}$ For the Fourier transform spectroscope solar observations the instrumental profile is better described by a sinc function, but this has a negligible impact on the calculated profiles.
} 

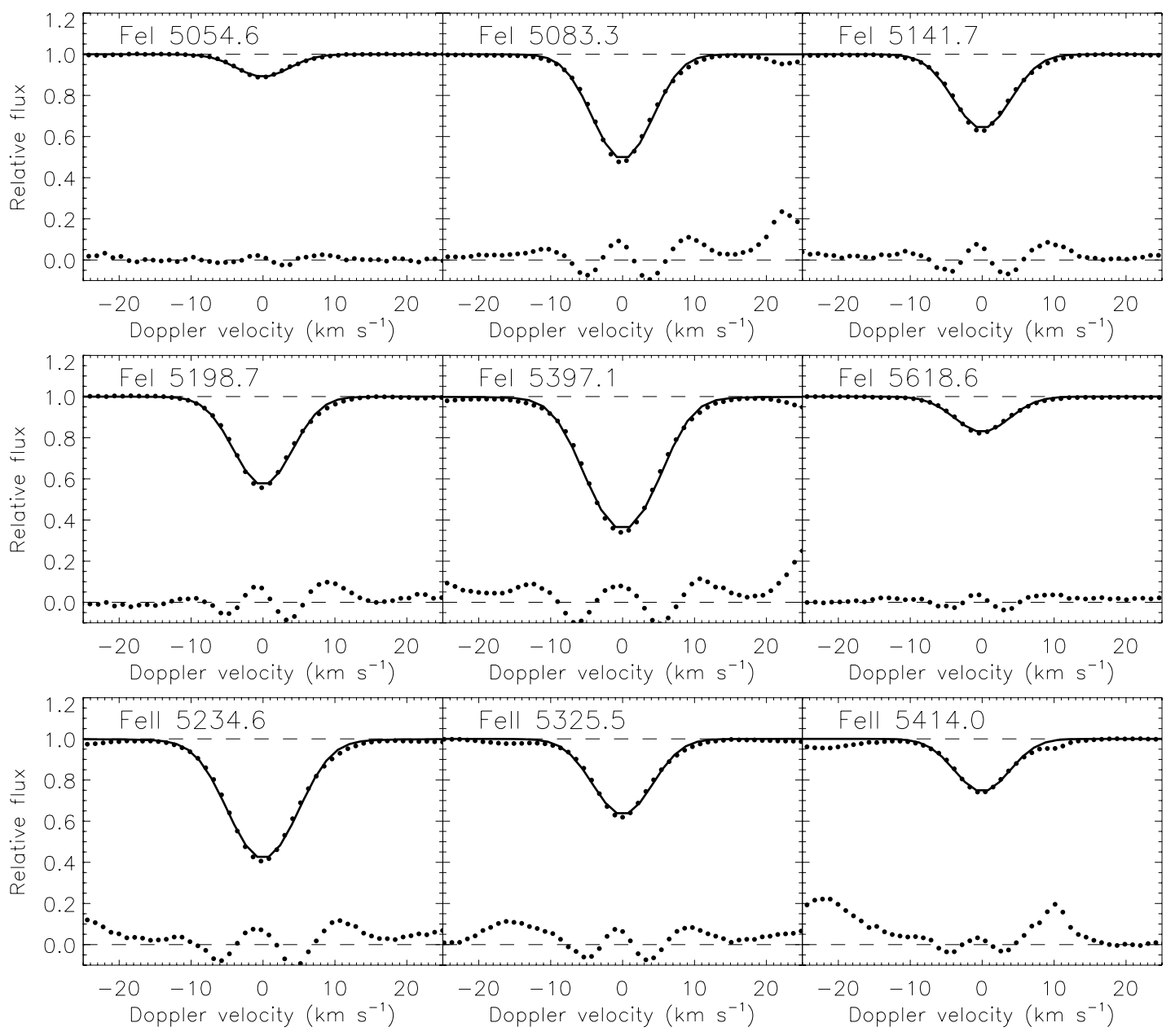

Fig. 8. - Comparison between the synthetic profiles calculated with the one-dimensional model atmosphere of Procyon (solid line) and the observations ( filled circles). The residuals (predicted and observed) are also shown, but multiplied by a factor of 5 .

tial as used by Gray (1981a). The scatter in $v_{\text {rot }} \sin i$ is clearly significantly larger with one-dimensional than with three-dimensional model atmospheres when using otherwise identical analyses. The reason why lines with $W_{\lambda} \simeq$ $70-100 \mathrm{~m} \AA$ apparently indicate the correct $v_{\text {rot }} \sin i$, while weaker lines do not, remains a mystery to us; in fact, the solar analysis shows the same effect (Fig. 11).

\subsection{Line Asymmetries and Shifts}

A major advantage with using three-dimensional hydrodynamical model atmospheres for spectral synthesis besides the elimination of free parameters like mixing length parameters, micro- and macroturbulence, is that the convective velocity field and temperature inhomogeneities are self-consistently computed. As a consequence, the imprint of convection in the form of asymmetries and shifts of spectral lines can be predicted and compared with observations. Furthermore, the predicted line asymmetries are on an absolute wavelength scale. Since different lines have different sensitivity to the photospheric structure and convective Doppler shifts, all lines show unique asymmetries.

Figure 12 illustrates the excellent agreement between predicted and observed line asymmetries for both $\mathrm{Fe} I$ and $\mathrm{Fe}$ II lines and weak and strong lines. Since the exact radial velocity of Procyon is unknown, the observed spectrum is not on an absolute wavelength scale. Therefore, the zero point for all observed bisectors in Figure 12 has been shifted by the same amount to make them coincide with the theoretical bisectors on average (see further discussion below and in $\S 8$ ). It should be noted, however, that the observed relative shifts between different lines shown in Figure 12 have been derived from the observations and are obviously well predicted by the three-dimensional calculations.

Although the general agreement between theory and observations is quite satisfactory, there are several notable exceptions for which the discrepant bisectors may signal problems like blends or an erroneous laboratory wavelength. In the case of $\mathrm{Fe}_{\mathrm{I}} \lambda 4745.8, \mathrm{Fe}_{\mathrm{I}} \lambda 5434.5$, and $\mathrm{Fe} \mathrm{I}$ $\lambda 4576.3$ the reason is most likely minor blends in the wings, which is supported by the good correspondence between predicted and observed bisectors for larger line depths. The $\mathrm{Fe}$ II $\lambda 4629.339$ line can be suspected to have an erroneous laboratory wavelength due to its uncharacteristically large offset of about $9 \mathrm{~mA}$ to the red. It is noteworthy that this line is the only Fe II line in our sample which is not included in the compilation of S. Johansson (1998, private communication) and the laboratory wavelength has instead been adopted from VALD.

The bisector comparison is summarized in Figure 13, which shows the difference between the theoretical and observed bisectors for the clean samples of $\mathrm{Fe}_{\mathrm{I}}$ and $\mathrm{Fe}$ II lines together with a few additional unblended $\mathrm{Fe}$ II lines 

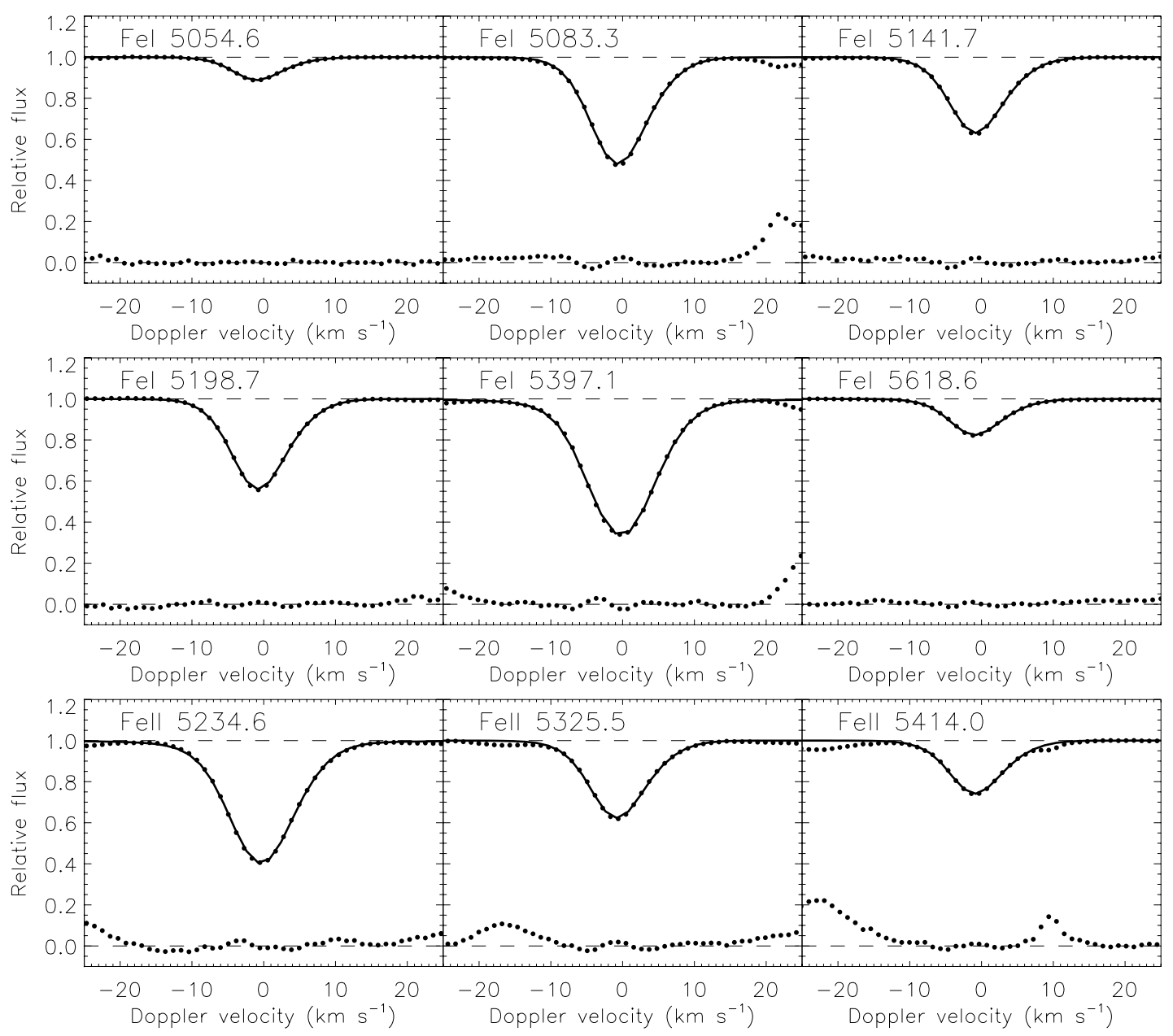

FIG. 9. - Comparison between the synthetic profiles calculated with the three-dimensional model atmosphere of Procyon and the observations ( filled circles). The residuals (predicted and observed) are also shown, but multiplied by a factor of 5 .

which lack an accurate determination of the transition probability and therefore are not included in the estimate of the $\mathrm{Fe}$ abundance presented below (see $\S 6$ ). For most lines, the predicted bisector is simply offset from the observed bisector, i.e., the difference is essentially a vertical line, without a trend with line depth that could signal an improper theoretical velocity field. The scatter around zero difference is, at least partly, due to errors in the laboratory wavelengths, as well as the finite $\mathrm{S} / \mathrm{N}$ and spectral resolution of the observations. Close to the continuum, the effects of weak blends become significant, which causes a larger scatter and in a few cases a deviating bisector. Typically, the shapes of the bisectors can be predicted to within $50 \mathrm{~m} \mathrm{~s}^{-1}$, while the typical velocity spans are $500 \mathrm{~m} \mathrm{~s}^{-1}$ or more, which is quite remarkable considering the sensitivity of line asymmetries upon the detailed atmospheric structure. In fact, the agreement is even slightly better than for the Sun (Asplund et al. 2000b).

A particularly interesting result arising from the line asymmetry comparison is the predicted blueward hook close to the continuum in the bisectors of some lines. All predicted Fe lines with $h<0.5$, and hence partly saturated, show this behavior, while it is not present in any weaker lines. The blue hook is induced by the influence of extended Lorentzian wings from the upflowing and hence blueshifted granules (Dravins 1990). As a result of the bias in line strength toward granules due to their steeper temperature gradients, lines tend to first saturate and develop damping wings there. The extended nature of these blueshifted wings can thus influence the resulting bisector shape, in particular close to the continuum. Even though blends tend to increase the uncertainty in the measured bisectors close to the continuum, there also exists observational evidence for this blueward hook. In particular, Fe I $\lambda 5415.2$ is a convincing case, but also $\mathrm{Fe}$ I $24903.3, \mathrm{Fe}_{\mathrm{I}}$ 25397.1, and $\mathrm{Fe}$ II $\lambda 4620.5$ support this conclusion. From the Procyon atlas of Griffin \& Griffin (1979), Dravins (1987) claimed to have identified a similar feature by averaging over many lines; however, the feature is not apparent in the average bisectors discussed in $\S 3.2$.

As expected from the good general agreement in terms of line asymmetries, the predicted differential line shifts match closely to the observations. Figure 14 shows the difference between predicted and observed line shifts for the $\mathrm{Fe} \mathrm{I}$ and $\mathrm{Fe}$ II lines; the $\mathrm{Fe}$ I $\lambda 4903.3101$ and $\mathrm{Fe}$ II $\lambda 4629.3390$ lines stand out, probably with erroneous laboratory wavelengths. Since the radial velocity of Procyon is unknown, the observed profiles have been shifted by $-1.56 \mathrm{~km} \mathrm{~s}^{-1}$ in order to make the difference between predictions and observations disappear on average. The mean of the differences between predicted and observed line shifts amounts to 0.00 $\mathrm{km} \mathrm{s}^{-1}$ with $\sigma=0.10 \mathrm{~km} \mathrm{~s}^{-1}$, implying that the radial 


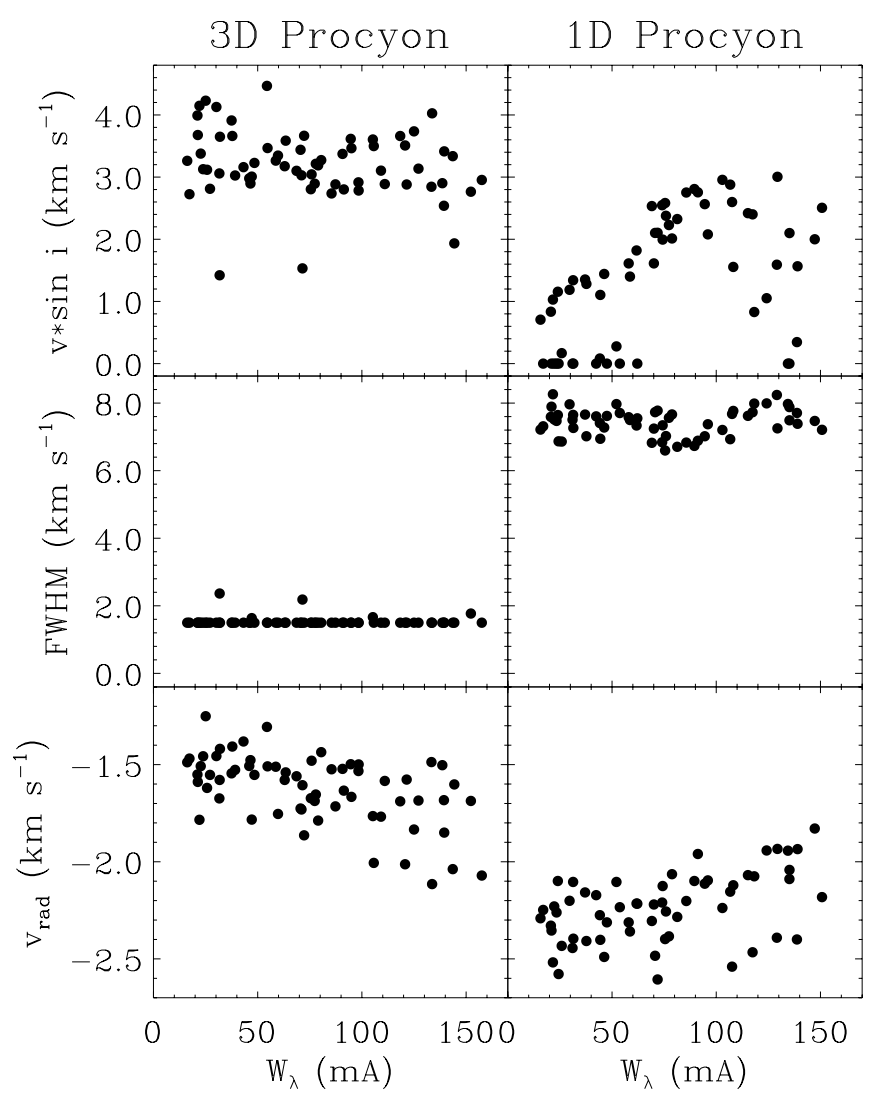

FIG. 10.-Optimal values for $v_{\text {rot }} \sin i$, the FWHM of a Gaussian profile convolved to the synthetic line profile, and the velocity shift $\left(v_{\mathrm{rad}}\right)$ to match the model and observed profiles of Procyon. The three panels on the left correspond to the three-dimensional model atmosphere, whereas the three on the right correspond to the one-dimensional model assuming a microturbulence of $2.2 \mathrm{~km} \mathrm{~s}^{-1}$.

velocity can be equally well determined in threedimensional analyses from fitting the whole profiles (Fig. $10)$ or only the line center. The observed weakening of the convective blueshift for stronger lines is the result of the disappearance of the convective inhomogeneities as the line formation region is gradually moved outward. Unfortunately, the present observations do not include sufficiently strong lines to determine if a plateau at zero convective line shifts exists, like that found for the Sun (Allende Prieto \& García López 1998), and the three-dimensional convection simulation cannot be invoked either to answer this question. As is clear from Figure 14, the predicted line shifts become systematically biased for stronger lines, which is not surprising given the likely departures from LTE in the cores of such lines. Besides, the three-dimensional simulations lose realism for the upper atmosphere as a result of the influence of the upper boundary and missing magnetic fields. In those layers, the presence of propagating waves and the fact that the energy balance is determined by a few strong lines out of LTE complicate quite seriously the modeling. A similar deficiency was detected in the solar study (Asplund et al. 2000b).

In Figure 15 we have mimicked $\mathrm{Fe}$ I lines of different strengths by altering the iron abundance in the calculations. Although a quantitative comparison is not possible because of the different ways in which the two plots were constructed, the general behavior of $\mathrm{Fe}$ I line asymmetries shown in Figure 5 is clearly reproduced by the theoretical

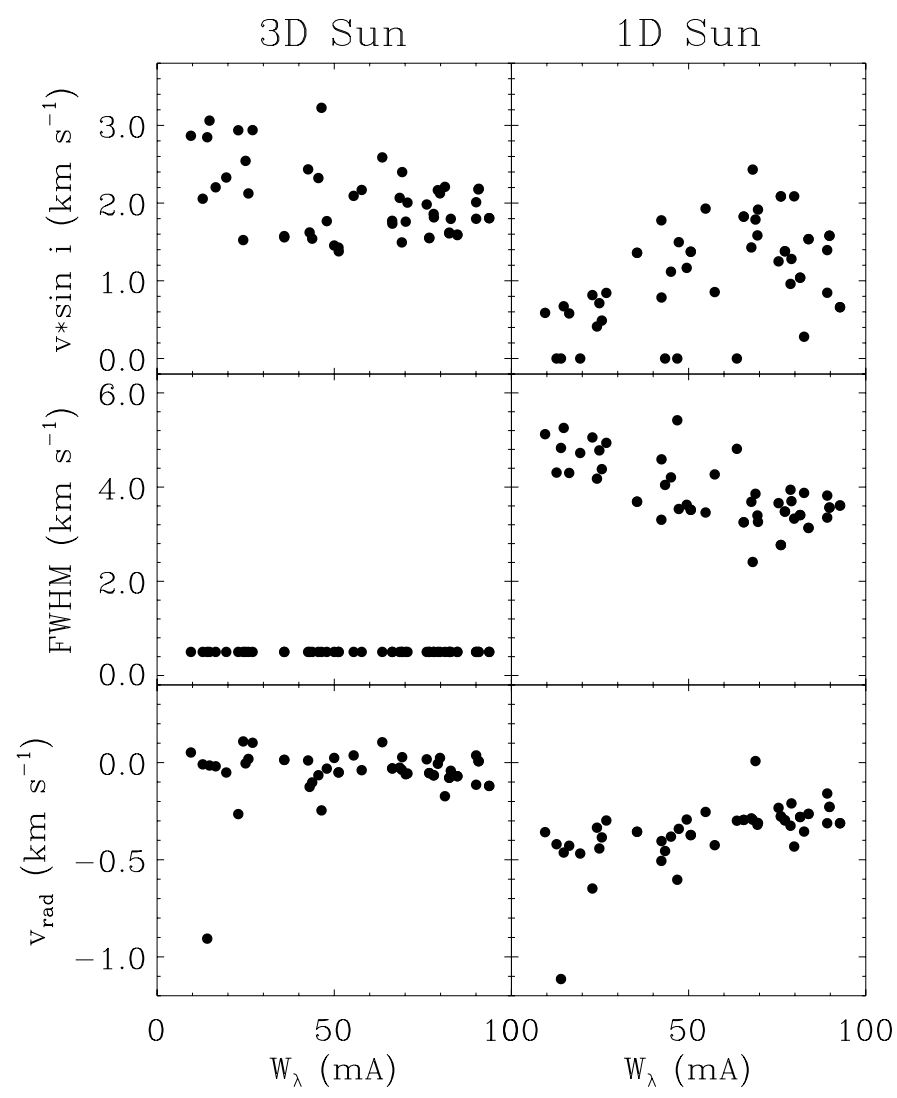

Fig. 11.-Optimal values for $v_{\text {rot }} \sin i$, the FWHM of a Gaussian profile convolved to the synthetic line profile, and the velocity $\operatorname{shift}\left(v_{\mathrm{rad}}\right)$ to match the model and observed profiles of the Sun. The three panels on the left correspond to the three-dimensional model atmosphere, whereas the three on the right correspond to the one-dimensional model.

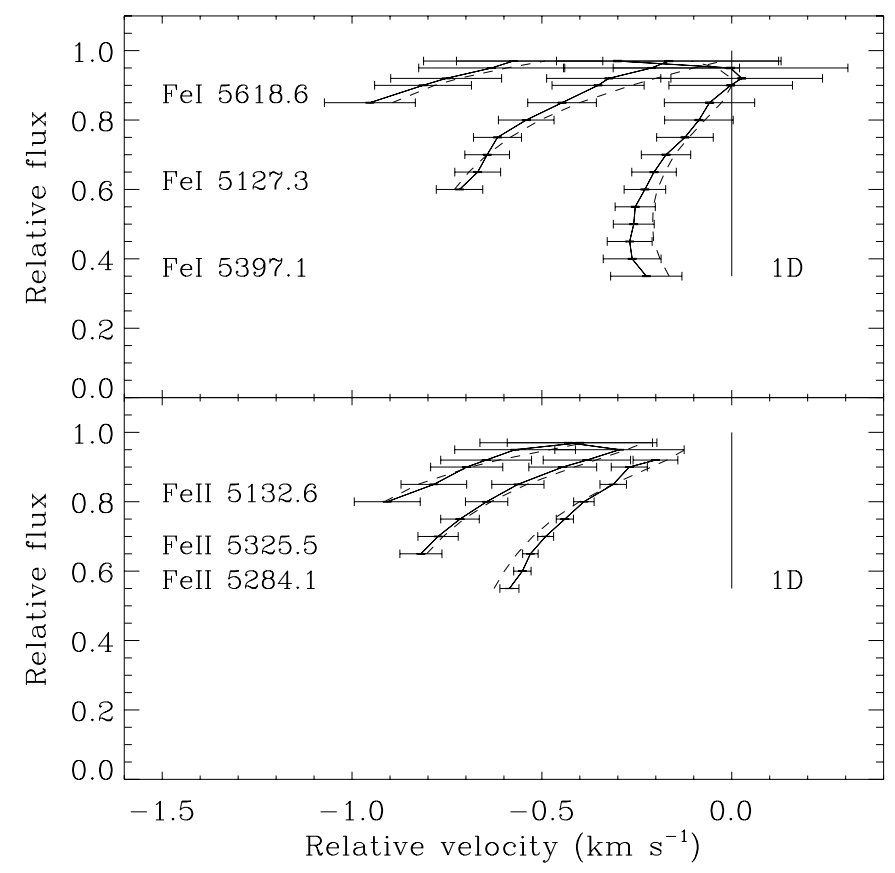

FIG. 12.-Comparison between line bisectors of three iron lines measured in the observed spectrum of Procyon (solid lines with error bars) with those measured in the synthetic spectrum calculated from the threedimensional model atmosphere (dashed lines). 


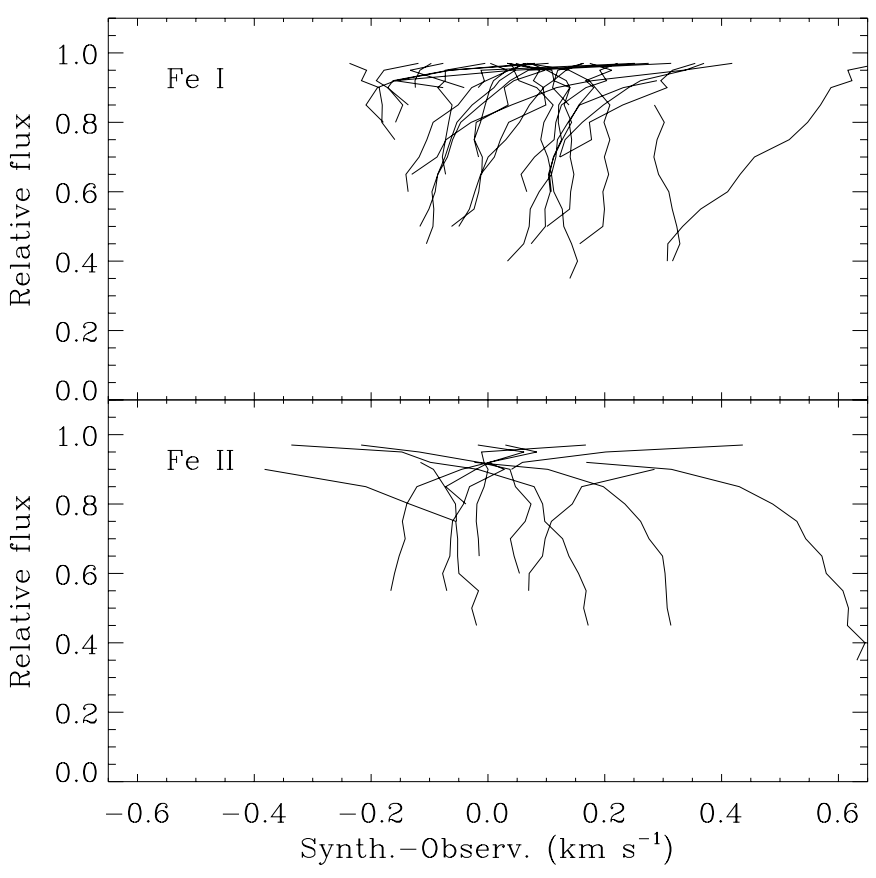

FIG. 13.-Difference between predicted and observed Fe I (upper panel) and $\mathrm{Fe}$ II (lower panel) bisectors.

calculations. We have calculated the line velocity shifts $(v)$ for a large group of $\mathrm{Fe} \mathrm{I}$ lines, and as Figure 16 reveals, the intrinsic scatter of the convective shifts for weak lines about a straight fit is significantly smaller in the Sun than in Procyon. This result confirms qualitatively the observational findings in $\S 3.1$. It should be noted, however, that since the same lines have not been used for the theoretical and observed distributions, one should not be surprised by minor differences in the results. The predicted slopes of the velocity shifts as a function of equivalent width are $5.9 \times 10^{-3}$ for the Sun and $6.0 \times 10^{-3}$ for Procyon, virtually identical. Neither of them are very close to the observed slopes, but the agreement is clearly better for Procyon, whose observed value is $4.9 \times 10^{-3}$, than for the Sun $\left(3.2 \times 10^{-3}\right.$; see Asplund et al. 2000a).

The line asymmetry comparison clearly shows that the adopted three-dimensional model atmosphere provides

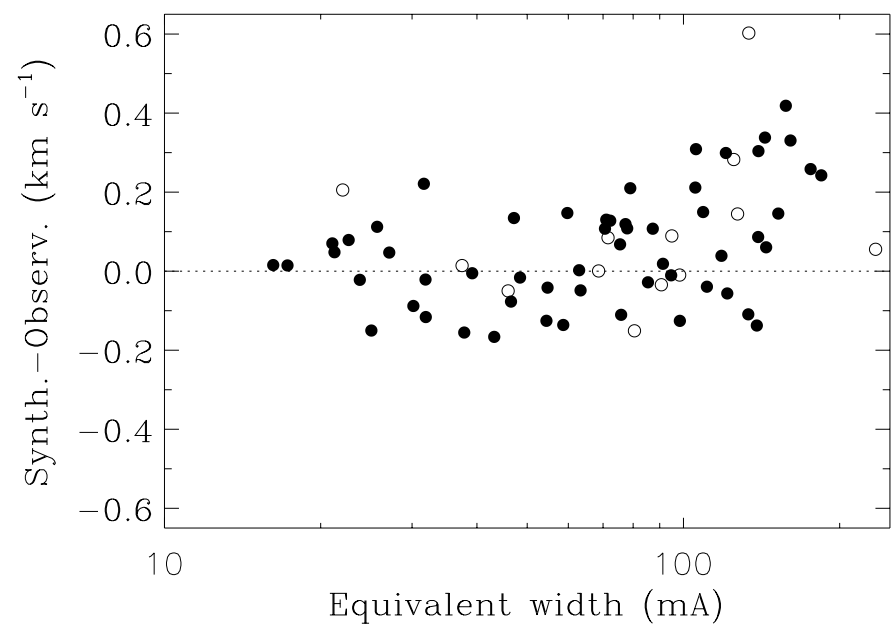

FIG. 14.-Difference between predicted and observed Fe I ( filled circles) and $\mathrm{Fe}$ II (open circles) line shifts.

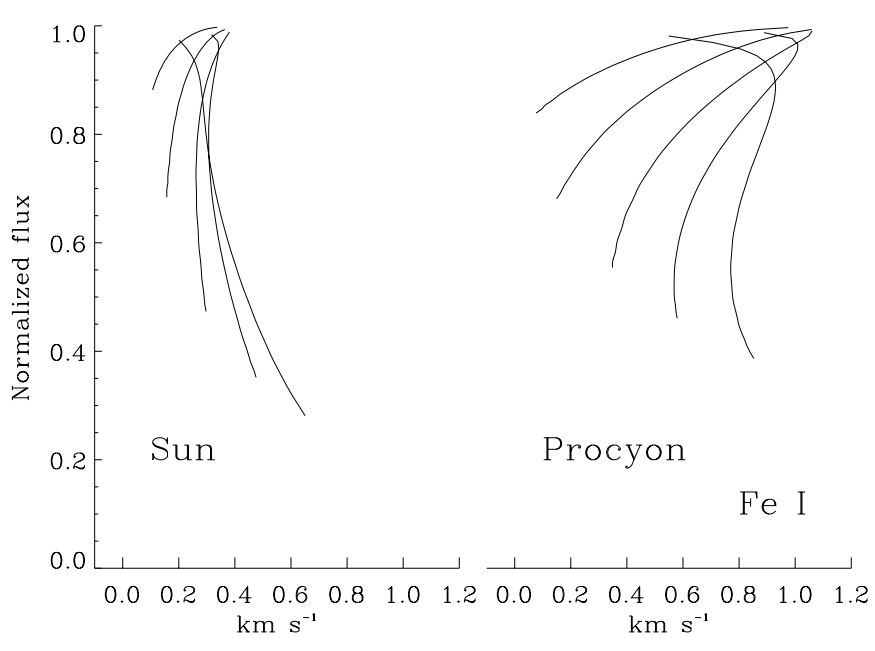

FIG. 15.-Average bisectors for theoretical $\mathrm{Fe} \mathrm{I}$ lines produced in the time-dependent hydrodynamical three-dimensional model atmosphere with a continuum-normalized flux at the line center $f: h<0.35$, $0.35<h<0.55,0.55<h<0.75$, and $h>0.75$. To simulate lines of different strength, we adopted a range of iron abundances in the calculation of a single line profile.

quite a realistic description of the granulation properties on Procyon. We stress again that one-dimensional models can predict neither the bisectors nor the shifts of lines.

\section{THE IRON ABUNDANCE OF PROCYON}

The chemical abundances inferred from the comparison between our spectroscopic observations and the threedimensional hydrodynamical model atmosphere of Procyon have a special interest, as this is the most common driver for stellar spectroscopy. It is particularly useful to refer our results to those from classical one-dimensional model atmospheres, on which most of the published stellar abundances are based. Here we determine the iron abundance of Procyon with the two types of models (see $\S 4.2$ ), making a strictly differential comparison between them.

\subsection{Classical One-Dimensional Models}

In addition to the free parameters in the $\chi^{2}$ analysis (iron abundance, $v_{\text {rot }} \sin i$, FWHM, and $v_{\text {rad }}$ ), one must also specify the microturbulence $\left(\xi_{\text {tur }}\right)$ in analyses based on onedimensional model atmospheres. Variations in this parameter affect both the line strengths and shapes, in particular for partly saturated lines. Following standard practice, we have carried out several $\chi^{2}$ analyses using different values of $\xi_{\text {tur }}$ in order to select the value which removes any trend in derived abundances with line strength. For Procyon, our choice of lines, transition probabilities, and model atmospheres suggests a value of $\xi_{\text {tur }}=2.2 \mathrm{~km} \mathrm{~s}^{-1}$. The results are displayed in the right-hand panels of Figure 17. The mean abundance derived from the neutral iron lines (filled circles) and ionized iron lines (open circles) is $\log \epsilon(\mathrm{Fe})=7.30 \pm 0.02 \quad(\sigma=0.11) \quad$ and $\quad 7.32 \pm 0.03$ $(\sigma=0.08)$ dex, respectively.

A similar analysis of the same lines used by Asplund et al. (2000c) in the disk-integrated solar atlas (Kurucz et al. 1984), but adopting the same sources for the radiative transition probabilities used for Procyon's $(\S 4.4)$, provided $\log \epsilon(\mathrm{Fe})_{\odot}=7.47 \pm 0.01(\sigma=0.07)$ dex for the Fe I lines and $\log \epsilon(\mathrm{Fe})_{\odot}=7.41 \pm 0.03(\sigma=0.11)$ dex for the Fe II lines with a MARCS model atmosphere and $\xi=1.0 \mathrm{~km}$ 

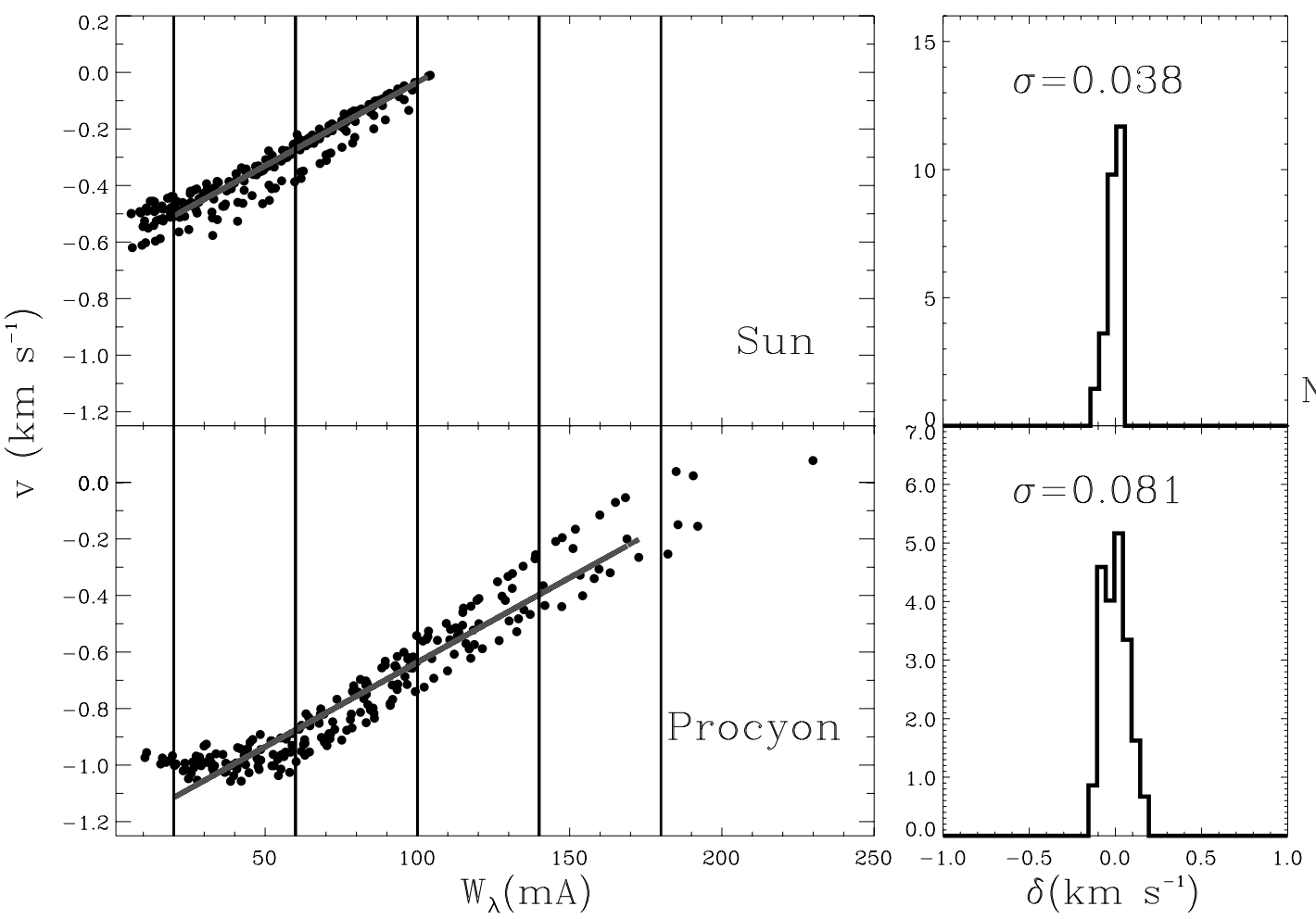

FIG. 16.-Left panels: Predicted velocity shifts between the stellar and laboratory wavelengths for relatively weak lines. Right panels: Difference between the measured shifts and the linear fits to lines with $20 \leq W_{\lambda} \leq 180 \mathrm{~m} \AA$ (histogram); the width of the distribution is quantified by the $\sigma$ of a Gaussian fit to the data.
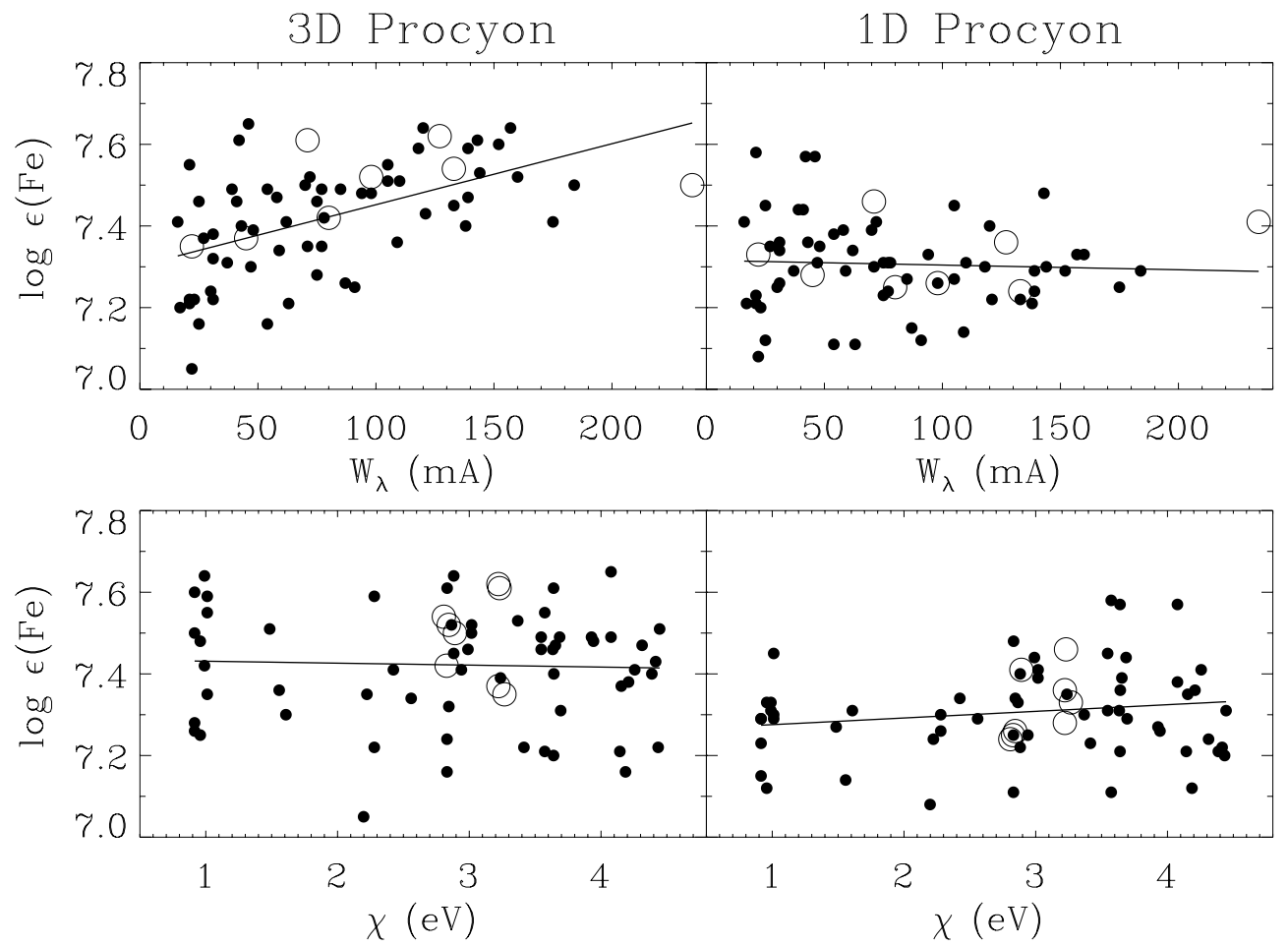

FIG. 17.- Iron abundance derived for Procyon from Fe I ( filled circles) and Fe II lines (open circles), as a function of the equivalent width (upper panels) and the line excitation potential (lower panels). 

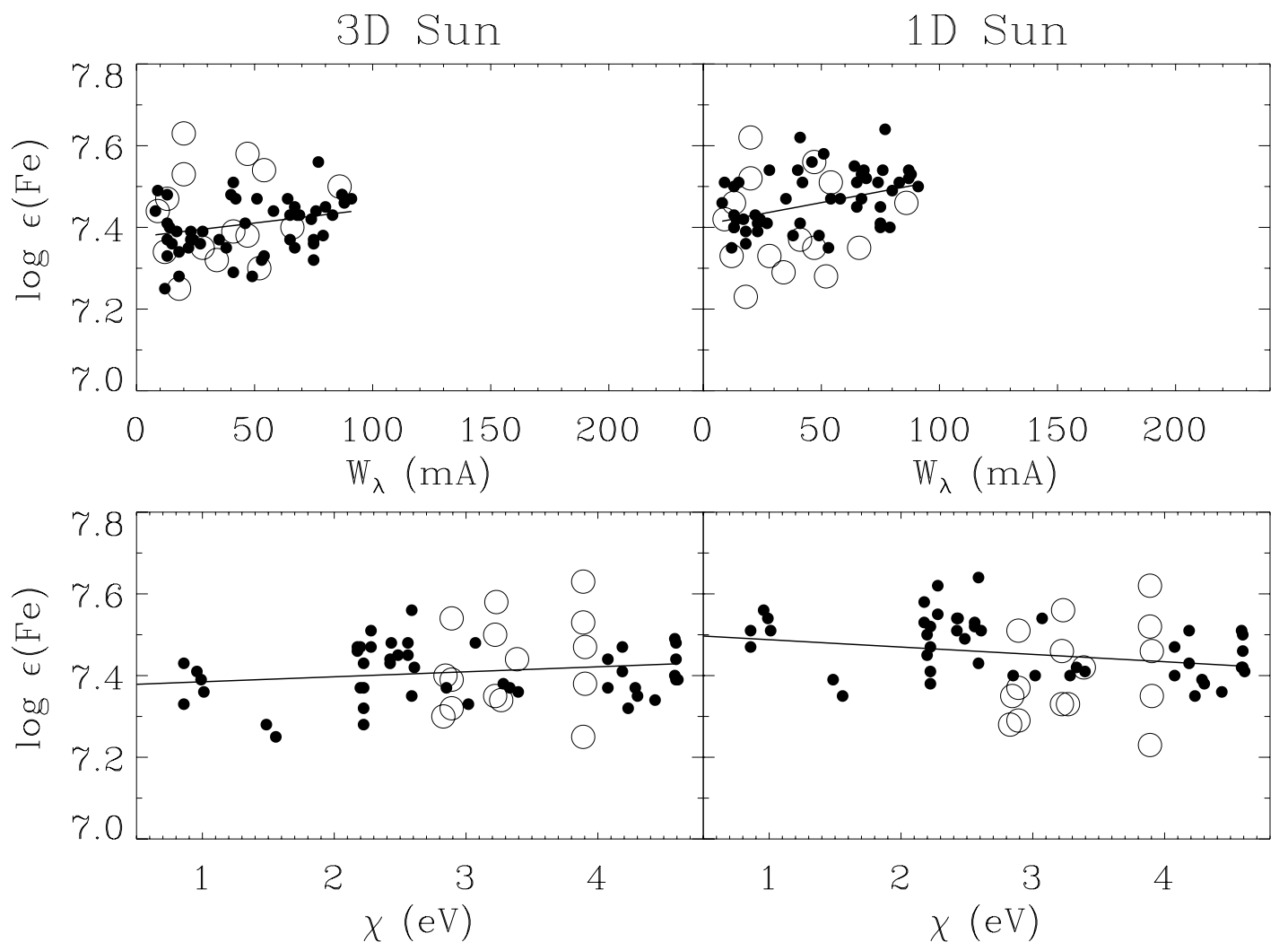

FIG. 18.- Iron abundance derived for the Sun from Fe I ( filled circles) and $\mathrm{Fe}$ in lines (open circles), as a function of the equivalent width (upper panels) and the line excitation potential (lower panels).

$\mathrm{s}^{-1}$. These figures are represented against equivalent width and excitation potential in the right-hand panels of Figure 18.

\subsection{Three-Dimensional Models}

Without an adjustable microturbulence parameter, the process of determining the abundances from the line profiles is straightforward from the $\chi^{2}$ analysis. To take advantage of all the information in the line profiles, and in parallel to our procedure for the one-dimensional models, we fit $v_{\text {rot }} \sin i, \mathrm{FWHM}$, and $v_{\text {rad }}$, for each individual line. Now, FWHM is also that of a Gaussian, but it only accounts for the instrumental profile, as macroturbulence is deemed unnecessary (§ 5.1). The left-hand panels of Figure 17 show the abundances from different lines plotted against the line equivalent width and the line excitation potential. The mean abundance is $\log \epsilon(\mathrm{Fe})_{\odot}=7.41 \pm 0.02(\sigma=0.14)$ dex for the neutral iron lines and $\log \epsilon(\mathrm{Fe})_{\odot}=7.49 \pm 0.04$ $(\sigma=0.10)$ dex for the singly ionized iron lines. The retrieved abundances tend to be higher for the stronger lines, as also found for the solar case with a more limited range of equivalent widths (see Fig. 18). This can be likely ascribed to the limited resolution of the simulations, which miss the highest convective velocities and therefore Doppler shifts. To put this into perspective, we note that this trend is similar to an underestimated microturbulence of about $0.3 \mathrm{~km} \mathrm{~s}^{-1}$ for Procyon, which highlights that it is a relatively minor shortcoming of the three-dimensional simulations as most of the needed convective Doppler shifts are already accounted for. Restricting the average to lines with equivalent widths less than or equal to $50 \mathrm{~m} \AA$, we obtain $7.35 \pm 0.03(\sigma=0.15 ; 22$ lines) and $7.36 \pm 0.01 \mathrm{dex}(\sigma=0.01$; two lines) for neutral and singly ionized iron lines, respectively.

The analysis of the selected solar flux lines previously used for the one-dimensional case, together with the $200 \times 200 \times 82$ three-dimensional simulation described by Asplund et al. (2000b), led to the following estimates of the solar iron abundance: $\log \epsilon(\mathrm{Fe})_{\odot}=7.40 \pm 0.01(\sigma=0.07)$ and $7.43 \pm 0.03(\sigma=0.11)$ dex, as derived from the neutral and singly ionized iron lines, respectively. These figures change very mildly when we restrict the sample to lines with equivalent widths smaller than $50 \mathrm{~m} \AA$, as all the lines considered have equivalent widths smaller than $100 \mathrm{~m} \AA$.

The small differences in derived abundances with those given in Asplund et al. (2000c), which are based on the same three-dimensional model atmosphere as employed here, can be traced to slightly different adopted transition probabilities $(\lessgtr 0.02 \mathrm{dex})$ and the use of flux profiles instead of disk center intensity profiles ( $\lesssim 0.02 \mathrm{dex})$. Our new estimates do not supersede the solar iron abundances given in Asplund et al. (2000c). They are only intended to enable a differential study of Procyon.

\subsection{Discussion}

Estimates of the accuracy of the $\log g f$ for the transitions used are readily available, either from O'Brian et al. (1991) or from the tables in the Appendix. We can use them to perform an analysis similar to that in $\S 3.1$ for the line velocity shifts. This reveals that the errors in the $\log g f$ values cannot account for more than about half of the scatter in the iron abundances. Even though three- 


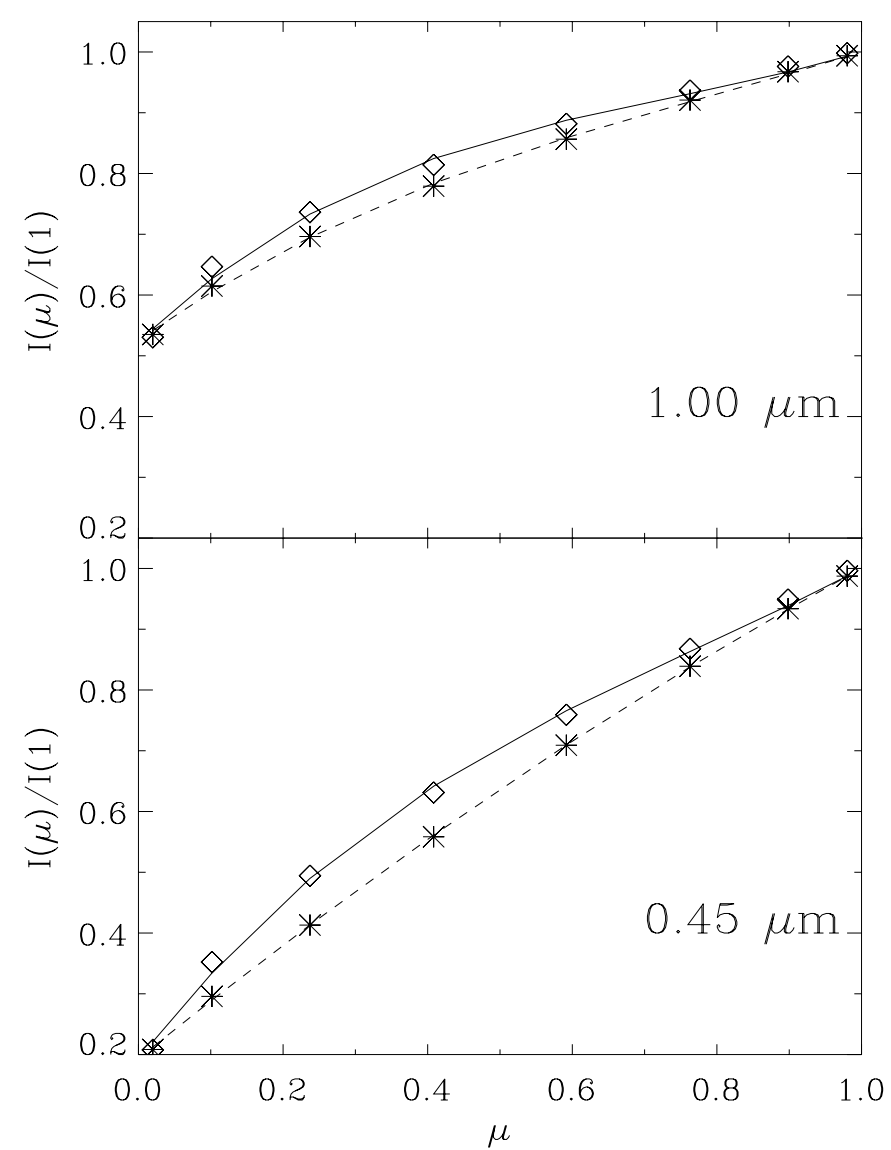

FIG. 19.- - Limb darkening for the three-dimensional (rhombi) and onedimensional (asterisks) models. Third-order polynomials have been fitted by regular least-squares fits to the data.

dimensional models need to be improved, there are probably other important error sources in our calculated line profiles. Departures from LTE are a likely candidate. We do not observe a larger scatter or a systematic deviation for low-excitation $\mathrm{Fe}$ I lines that one could expect from the three-dimensional NLTE calculations of Shchukina \& Trujillo Bueno (2001), but we notice a significantly larger scatter for the iron abundance determined from $\mathrm{Fe}$ I lines in Procyon than in the Sun, and departures from LTE are expected to grow for warmer stars. Because of the shown imperfections in the three-dimensional models, the preferred iron abundance for Procyon is that derived from the three-dimensional analysis of weak lines: $\log \epsilon(\mathrm{Fe})_{\odot}=7.36$ $\pm 0.03(\sigma=0.15)$ dex.

The main conclusions of our study of the iron abundance are as follows:

1. The abundances derived from $\mathrm{Fe} \mathrm{I}$ and $\mathrm{Fe}$ II lines are consistent for both one-dimensional and three-dimensional analyses, and with a higher coherence for the threedimensional model. This implies that departures from LTE, which should be minimal for Fe II, are likely small. Nevertheless, they could be responsible for a significant fraction of the scatter between the abundances retrieved from different lines, providing a plausible explanation for the larger scatter observed for Procyon than for the Sun.

2. The differences between the iron abundances derived from one-dimensional and three-dimensional analyses (setting aside lines stronger than $50 \mathrm{~m} \AA$ in the case of Procyon) are small ( $\lesssim 0.05 \mathrm{dex})$, and the same conclusion applies to the solar case. Other stars and elements may show larger differences.

3. Procyon is marginally deficient in iron compared to the Sun by about 0.05 dex.

\section{CENTER-TO-LIMB VARIATION}

One of the applications of model atmospheres is to derive limb-darkening laws. These are required, for example, to correct interferometric measurements of stellar angular diameters and affect directly the otherwise fully empirical calibrations of effective temperature against color indices (see, e.g., Mozurkewich et al 1991).

Here we compare the center-to-limb variation predicted by homogeneous models and the three-dimensional simulations. Taking the emerging intensity for different angles at all different spatial locations, we produce a spatially and time-averaged intensity for different ray inclinations. Figure 19 shows the predicted limb darkening for the onedimensional and three-dimensional models. Two wavelengths were selected, 4500 and $10000 \AA$ A. Strong differences are obvious in the plot, the limb darkening being markedly nonlinear for the inhomogeneous model. We have fitted the data to third-order polynomials by regular least-squares fits (see eq. [6] in Hanbury Brown, Davis, \& Allen 1974), and the results correspond to the solid (three-dimensional) and dashed (one-dimensional) lines in Figure 19. Lower order polynomials were inappropriate for the three-dimensional model atmosphere. Hanbury Brown et al. (1974) calculated the interferometric correlation factor associated with this particular limb-darkening law. At $4500 \AA$, their determined angular diameter for a uniformly emitting disk should be corrected by factors of 1.081 and 1.064 for the onedimensional and three-dimensional cases, respectively. The one-dimensional correction obtained from an older version of MARCS (Gustafsson et al. 1975) used by Mozurkewich et al. (1991) was 1.07 at $4500 \AA$. The radius and the effective temperature of Procyon we derived in $\S 4.1$ should therefore be slightly corrected from 2.071 to $2.059 R_{\odot}$ and from 6512 to $6530 \mathrm{~K}$, respectively. The correction between threedimensional and one-dimensional models, which amounts to roughly $1.6 \%$, implies a correction to the effective temperature of roughly $50 \mathrm{~K}$ for a star like Procyon A, showing the importance of detailed model atmospheres for establishing a truly empirical $T_{\text {eff }}$ scale from measurements of angular diameters (see, e.g., di Benedetto 1998).

\section{ABSOLUTE RADIAL VELOCITY}

When discussing measurements of stellar radial velocities, it is commonly assumed that an observed shift of the spectral features from their wavelengths at rest can be directly translated to a velocity projected along the line of sight. Lindegren, Dravins, \& Madsen (1999) have recently discussed the dangers in doing so, especially when it comes to precise measurements, pointing out several effects that may need to be considered.

The gravitational shift is proportional to the gravitational field, $V_{g}=G M /(R c)$, and for late-type dwarfs amounts to about $0.5 \mathrm{~km} \mathrm{~s}^{-1}$. In the case of Procyon A, we have a special advantage, as its mass, radius, and proper motion are well determined. The gravitational redshift, including the blueshift induced by Earth, is $V_{g}=0.436$ $\pm 0.019 \mathrm{~km} \mathrm{~s}^{-1}$, where we have used the corrected stellar radius $R=2.06 \pm 0.02 R_{\odot}($ see $\S 7$ ). The transverse Doppler 
effect scales with the square of the modulus of the velocity, $V_{\mathrm{tD}}=\left|V_{t}\right|^{2} /(2 c)$, and becomes significant, reaching $\sim 0.25$ $\mathrm{km} \mathrm{s}^{-1}$ for the fastest halo stars orbiting at $\sim 400 \mathrm{~km} \mathrm{~s}^{-1}$. The proper motion of Procyon as measured by Hipparcos corresponds to a velocity perpendicular to the line of sight of $V_{t}=20.87 \pm 0.07 \mathrm{~km} \mathrm{~s}^{-1}$, which translates to a negligible transverse Doppler shift of less than $1 \mathrm{~m} \mathrm{~s}^{-1}$.

The list of contributors to the observed line shifts that have little to do with the star's space motion is much longer: pulsations, stellar winds, surface convection, pressure gradients, etc. In the case of quiet late-type dwarfs, stellar winds are not expected to disturb the photospheric line wavelengths, and the integrated light pulsations are known to have periods of minutes and amplitudes of a few meters per second or less, as detected in the Sun (see, e.g., Fossat \& Ricort 1975; Claverie et al. 1979) or Procyon itself (Martić et al. 1999). Pressure gradients have been shown to typically produce line asymmetries and shifts of only a few meters per second for solar-like stars (Allende Prieto, García López, \& Trujillo Bueno 1997). In conclusion, the main disturbing element is the velocity pattern of convective origin. The detailed three-dimensional hydrodynamical simulations presented here provide an accurate description of the convective velocity-temperature fields in the photosphere and therefore constitute a means to zero the photospheric velocities. Confronting the measured line shifts with the predictions of the three-dimensional model (see $\S 5.2$ ) and restricting the analysis to lines weaker than $100 \mathrm{~mA}$, we determine a velocity shift of the observed spectrum of Procyon A by $-1.56 \pm 0.13 \mathrm{~km} \mathrm{~s}^{-1}$. That amount has to be divided between the remaining effects expected to be important in the case of Procyon A: gravitational redshift, orbital velocity, and systemic velocity.

Combining the astrometric orbital elements of Girard et al. (2000) with the radial velocity amplitude measured by Irwin et al. (1992), $1.401 \pm 0.093$ (from absolute measurements) or $1.482 \pm 0.110 \mathrm{~km} \mathrm{~s}^{-1}$ (from relative determinations), we find that the orbital velocity of Procyon A changed by less than $0.0004 \mathrm{~km} \mathrm{~s}^{-1}$ during the 2 days the observations were carried out, and its value was 1.115 (from absolute measurements) or $1.180 \mathrm{~km} \mathrm{~s}^{-1}$ (from relative determinations). Adopting $1.15 \pm 0.10 \mathrm{~km} \mathrm{~s}^{-1}$, we obtain for the heliocentric radial velocity of the system $V_{r}=-1.56$ $( \pm 0.13)-0.44( \pm 0.02)-1.15( \pm 0.10)=-3.15 \pm 0.17 \mathrm{~km}$ $\mathrm{s}^{-1}$.

\section{SUMMARY AND CONCLUSIONS}

We have observed the spectrum of Procyon A (F5 IV) from 4559 to $5780 \AA$ with an $\mathrm{S} / \mathrm{N}$ of $\sim 10^{3}$ and a resolving power of $2 \times 10^{5}$. We have measured the line bisectors and relative line shifts of a large number of $\mathrm{Fe}_{\mathrm{I}}$ and $\mathrm{Fe}$ II lines. Compared to the Sun, the convective velocity shifts of the lines are significantly larger and show a larger scatter from a linear relationship against equivalent width. The difference between the weakest and the strongest lines measured can reach up to $1 \mathrm{~km} \mathrm{~s}^{-1}$, which is almost twice as large as the range observed in the Sun. Among other interesting consequences, these large differential velocities are likely to limit the accuracy in the determination of absolute radial velocities in F-type stars.

We have constructed a new three-dimensional, timedependent hydrodynamical model atmosphere and tested it against the observed spectrum. The new model reproduces in detail most of the observed features, but we identify some room for improvement. At all levels, the comparison with the observed spectral lines shows a significant improvement compared to classical homogeneous models. The predicted line shifts and asymmetries are reasonably close to the observed ones. Our study of the iron abundance in the photosphere of Procyon reveals the following:

1. The abundances derived from $\mathrm{Fe} \mathrm{I}$ and $\mathrm{Fe}$ II lines are consistent for both one-dimensional and three-dimensional analyses, and to a higher degree for the latter. This implies that departures from LTE, which should be minimal for Fe II, are relatively small.

2. The differences between the mean iron abundances derived from one-dimensional and three-dimensional analyses are small $(\lesssim 0.05 \mathrm{dex})$.

3. We find the iron abundance of Procyon to be $\log \epsilon(\mathrm{Fe})=7.36 \pm 0.03(\sigma=0.15)$ dex, concluding, from a differential study of the solar spectrum, that the star is marginally deficient in iron compared to the Sun by $\sim 0.05$ dex.

We notice a scatter in the abundances from different lines that clearly exceeds the expectations from errors in the transition probabilities. Known imperfections in the threedimensional model should account for part of the excess errors. Departures from LTE are a potentially important source of error. Three-dimensional models with improved resolution and detailed NLTE calculations are needed. In addition, other deficiencies in the input physics could contribute and could be identified through meticulous tests against observations.

The detailed line shapes predicted by the model provide a reliable upper limit for the small projected rotational velocity of the star, $v_{\text {rot }} \sin i \leq 3.16 \mathrm{~km} \mathrm{~s}^{-1}$, with the correct value probably close to $2.7 \mathrm{~km} \mathrm{~s}^{-1}$. The three-dimensional model atmosphere shows a limb-darkening law that is noticeably different from the predictions of one-dimensional models. This finding has limited impact on the interferometric determination of the angular diameter of Procyon $(\sim 2 \%)$ and its effective temperature $(\sim 50 \mathrm{~K})$ but is obviously important in establishing an absolute scale of $T_{\text {eff }}$ based on direct measurements. The ability of the hydrodynamical model atmosphere to predict the convective shifts of the spectral lines, combined with the accurate parallax and proper motions determined by Hipparcos, the published orbital elements, and a careful assessment of the gravitational redshift, makes it possible to determine the space motion of the Procyon binary system within $0.17 \mathrm{~km} \mathrm{~s}^{-1}$.

We are indebted to the staff at McDonald Observatory, in particular David Doss, for invaluable assistance in the data acquisition. Sveneric Johansson and Ward Whaling kindly delivered line wavelengths and experimental $\mathrm{Fe}$ II $f$-values, respectively. Åke Nordlund, Robert Stein, and Regner Trampedach are thanked for expert help with the three-dimensional convection simulations. National Solar Observatory/Kitt Peak FTS data used here were produced by NSF/NOAO. We have made use of the Hipparcos Catalogue, NASA's Astrophysics Data System Abstract Service, the SIMBAD database at the CDS, and VALD. This research has been partially supported by the NSF (grant AST-0086321), the Robert A. Welch Foundation of Houston, Texas, the Swedish Natural Science Foundation (grant NFR F990/1999), and the Royal Swedish Academy of Sciences. 


\section{APPENDIX}

\section{A COMPILATION OF LABORATORY ABSOLUTE TRANSITION PROBABILITIES FOR Fe II LINES WITH $\lambda>300 \mathrm{~nm}$}

The experimental technique most widely used combines measured branching ratios and lifetimes of the upper levels to derive transition probabilities. Measurements of branching ratios of high quality have been published by Kroll \& Kock (1987), Whaling (as included in the compilation by J. R. Fuhr, G. A. Martin, \& W. L. Wiese 1988, private communication), Heise \& Kock (1990), and Pauls, Grevesse, \& Huber (1990). Level lifetimes have been derived with accuracies in the range 0.02-0.14 ns by Biemont et al. (1991), Guo et al. (1992), and Schnabel, Kock, \& Holweger (1999) using laser-induced fluorescence. Available lifetimes have been compiled and combined in Table 3. When more than one measurement was available, we averaged them out. The variation among the lifetime of the levels within a term is remarkably small (except maybe for the ${ }^{4} F$ term), as expected if LS coupling holds. Lower accuracy measurements that include ${ }^{4} D_{1 / 2}$ and ${ }^{4} F_{3 / 2}$ levels (providing for them $2.9 \pm 0.2$ and $3.7 \pm 0.2$, respectively) support the use of the term average values for those cases $(2.95 \pm 0.03$ and $3.70 \pm 0.15)$. The branching fractions measured by Kroll \& Kock (1987), Whaling, Heise \& Kock (1990), and Pauls et al. (1990) were converted to transition probabilities using different sources for the lifetimes of the involved upper levels. The third through sixth columns of Table 4 list their published $\log g f$ values, the quoted uncertainties, and the lifetimes used for the different transitions. We have rescaled the individual $\log g f$ values using the adopted lifetimes of Table 3 , to produce a weighted averaged when more than a single measure was available. When inconsistent $\log g f$ values were derived from Kroll \& Kock (1987) and Heise \& Kock (1990), the latter were preferred, following Schnabel et al. (1999). The finally adopted $\log g f$ values and their formal uncertainties are displayed in the last column of Table 4.

TABLE 3

RADIATIVE LIFETIMES FOR THE DIFFERENT LeVELS OF Fe II

\begin{tabular}{|c|c|c|c|c|c|c|}
\hline Term & $J$ & $\begin{array}{l}\text { Biemont et al. (1991) } \\
\text { (ns) }\end{array}$ & $\begin{array}{c}\text { Guo et al. (1992) } \\
\text { (ns) }\end{array}$ & $\begin{array}{l}\text { Schnabel et al. (1999) } \\
\text { (ns) }\end{array}$ & $\begin{array}{l}\text { Adopted } \\
\text { (ns) }\end{array}$ & $\begin{array}{c}\text { Term Average } \\
\text { (ns) }\end{array}$ \\
\hline \multirow{5}{*}{${ }^{6} D \ldots \ldots$} & $9 / 2$ & $3.70 \pm 0.06$ & $\ldots$ & $\ldots$ & $3.70 \pm 0.06$ & $\ldots$ \\
\hline & $7 / 2$ & $3.68 \pm 0.07$ & $\ldots$ & $3.64 \pm 0.09$ & $3.66 \pm 0.07$ & $\ldots$ \\
\hline & $5 / 2$ & $3.63 \pm 0.08$ & $\ldots$ & $3.70 \pm 0.05$ & $3.68 \pm 0.05$ & $\ldots$ \\
\hline & $3 / 2$ & $3.83 \pm 0.10$ & $\ldots$ & $3.73 \pm 0.07$ & $3.76 \pm 0.07$ & $\ldots$ \\
\hline & $1 / 2$ & $3.76 \pm 0.10$ & $\ldots$ & $\ldots$ & $3.76 \pm 0.10$ & $3.71 \pm 0.05$ \\
\hline \multirow[t]{5}{*}{${ }^{6} F \ldots \ldots$} & $11 / 2$ & $3.19 \pm 0.04$ & $\ldots$ & $\ldots$ & $3.19 \pm 0.04$ & $\ldots$ \\
\hline & $9 / 2$ & $3.24 \pm 0.06$ & $\ldots$ & $\ldots$ & $3.24 \pm 0.06$ & $\ldots$ \\
\hline & $7 / 2$ & $3.26 \pm 0.10$ & $\ldots$ & $\ldots$ & $3.26 \pm 0.10$ & $\ldots$ \\
\hline & $5 / 2$ & $3.33 \pm 0.09$ & $\ldots$ & $\ldots$ & $3.33 \pm 0.09$ & $\ldots$ \\
\hline & $3 / 2$ & $3.34 \pm 0.10$ & $\ldots$ & $\ldots$ & $3.34 \pm 0.10$ & $3.27 \pm 0.06$ \\
\hline \multirow[t]{3}{*}{${ }^{6} P \ldots \ldots$} & $7 / 2$ & $3.73 \pm 0.05$ & $3.73 \pm 0.06$ & $\ldots$ & $3.73 \pm 0.05$ & $\ldots$ \\
\hline & $5 / 2$ & $3.83 \pm 0.07$ & $3.79 \pm 0.12$ & $\ldots$ & $3.81 \pm 0.07$ & $\ldots$ \\
\hline & $3 / 2$ & $\ldots$ & $3.71 \pm 0.12$ & $\ldots$ & $3.71 \pm 0.12$ & $3.75 \pm 0.05$ \\
\hline \multirow[t]{2}{*}{${ }^{4} P \ldots \ldots$} & $5 / 2$ & $\ldots$ & $3.43 \pm 0.09$ & $\ldots$ & $3.43 \pm 0.09$ & $\ldots$ \\
\hline & $3 / 2$ & $\ldots$ & $3.44 \pm 0.11$ & $\ldots$ & $3.44 \pm 0.11$ & $3.44 \pm 0.01$ \\
\hline \multirow[t]{4}{*}{${ }^{4} D \ldots \ldots$} & $7 / 2$ & $\ldots$ & $3.02 \pm 0.07$ & $2.97 \pm 0.02$ & $2.97 \pm 0.02$ & $\ldots$ \\
\hline & $5 / 2$ & $\ldots$ & $3.10 \pm 0.08$ & $2.90 \pm 0.06$ & $2.97 \pm 0.06$ & $\ldots$ \\
\hline & $3 / 2$ & $\ldots$ & $\ldots$ & $2.91 \pm 0.09$ & $2.91 \pm 0.09$ & $\ldots$ \\
\hline & $1 / 2$ & $\ldots$ & $\ldots$ & $\ldots$ & $2.95 \pm 0.03^{\mathrm{a}}$ & $2.95 \pm 0.03$ \\
\hline \multirow[t]{4}{*}{${ }^{4} F \ldots \ldots$} & $9 / 2$ & $\ldots$ & $3.87 \pm 0.09$ & $\ldots$ & $3.87 \pm 0.09$ & $\ldots$ \\
\hline & $7 / 2$ & $\ldots$ & $3.63 \pm 0.11$ & $\ldots$ & $3.63 \pm 0.11$ & $\ldots$ \\
\hline & $5 / 2$ & $\ldots$ & $3.75 \pm 0.14$ & $3.55 \pm 0.08$ & $3.60 \pm 0.08$ & $\ldots$ \\
\hline & $3 / 2$ & $\ldots$ & $\ldots$ & $\ldots$ & $3.70 \pm 0.15^{\mathrm{a}}$ & $3.70 \pm 0.15$ \\
\hline
\end{tabular}

${ }^{\text {a }}$ Measurement not available. The term average is adopted. Note that Hannaford et al. 1992 give $2.9 \pm 0.2\left({ }^{4} D\right)$ and $3.7 \pm 0.2$ $\left({ }^{4} F\right)$. 
TABLE 4

Oscillator Strengths For Fe il Lines IN the Optical Region

\begin{tabular}{|c|c|c|c|c|c|c|}
\hline $\begin{array}{l}\text { Wavelength } \\
\text { (̊̊) }\end{array}$ & Term & Kroll \& Kock (1987) & Whaling & Heise \& Kock (1990) & Pauls et al. (1990) & Adopted \\
\hline $3002.64 \ldots \ldots$ & ${ }^{4} P_{5 / 2}$ & $-0.93 \pm 0.07,3.9$ & $\ldots$ & $\ldots$ & $\ldots$ & $-0.87 \pm 0.07$ \\
\hline $3163.09 \ldots \ldots$ & ${ }^{4} F_{5 / 2}$ & $-2.82 \pm 0.07,4.0$ & $\ldots$ & $-2.82 \pm 0.07,4.0^{\mathrm{a}}$ & $\ldots$ & $-2.78 \pm 0.07$ \\
\hline $3170.34 \ldots \ldots$ & ${ }^{4} D_{1 / 2}$ & & $\ldots$ & $\ldots$ & $-2.49 \pm 0.08,3.4$ & $-2.42 \pm 0.08$ \\
\hline $3183.11 \ldots \ldots$ & ${ }^{4} F_{5 / 2}$ & $-2.10 \pm 0.07,4.0$ & $-2.04 \pm 0.11,4.0$ & $\ldots$ & $\ldots$ & $-2.04 \pm 0.06$ \\
\hline $3185.31 \ldots \ldots$ & ${ }^{4} F_{3 / 2}$ & $-2.78 \pm 0.07,4.1$ & $\ldots$ & $\ldots$ & $\ldots$ & $-2.74 \pm 0.07$ \\
\hline $3186.74 \ldots \ldots$ & ${ }^{4} D_{3 / 2}$ & $-1.67 \pm 0.04,3.2$ & $-1.71 \pm 0.11,3.4$ & $\ldots$ & $\ldots$ & $-1.63 \pm 0.04$ \\
\hline $3192.92 \ldots \ldots$ & ${ }^{4} D_{5 / 2}^{3 / 2}$ & $-1.95 \pm 0.11,3.2$ & $\ldots$ & $\cdots$ & $-1.92 \pm 0.07,3.4$ & $-1.88 \pm 0.06$ \\
\hline $3193.80 \ldots \ldots$ & ${ }^{4} D_{1 / 2}$ & $-\ldots$ & $\ldots$ & $\ldots$ & $-1.75 \pm 0.07,3.4$ & $-1.69 \pm 0.07$ \\
\hline $3196.08 \ldots \ldots$ & ${ }^{4} F_{7 / 2}$ & $-1.73 \pm 0.11,3.9$ & $-1.66 \pm 0.11,3.9$ & $\ldots$ & $\ldots$ & $-1.67 \pm 0.08$ \\
\hline $3210.45 \ldots \ldots$ & ${ }^{4} D_{3 / 2}$ & $-1.69 \pm 0.04,3.2$ & $-1.79 \pm 0.11,3.4$ & $-1.69 \pm 0.04,3.2^{\mathrm{a}}$ & $\ldots$ & $-1.66 \pm 0.04$ \\
\hline $3213.31 \ldots \ldots$ & ${ }^{4} D_{5 / 2}^{3 / 2}$ & $-1.27 \pm 0.07,3.2$ & $\ldots$ & $\ldots$ & $-1.31 \pm 0.07,3.4$ & $-1.25 \pm 0.05$ \\
\hline $3227.73 \ldots \ldots$ & ${ }^{4} D_{7 / 2}$ & $-1.06 \pm 0.11,3.7$ & $-1.13 \pm 0.04,3.7$ & $\ldots$ & $\ldots$ & $-1.02 \pm 0.04$ \\
\hline $3255.89 \ldots \ldots$ & ${ }^{6} D_{7 / 2}$ & $-2.52 \pm 0.04,4.2$ & $-2.50 \pm 0.22,4.0$ & $-2.52 \pm 0.04,4.2^{\mathrm{a}}$ & $\ldots$ & $-2.46 \pm 0.04$ \\
\hline $3277.35 \ldots \ldots$ & ${ }^{6} D_{9 / 2}$ & $-2.30 \pm 0.04,3.9$ & $-2.47 \pm 0.11,3.9$ & $\ldots$ & $\ldots$ & $-2.31 \pm 0.04$ \\
\hline $322.774 \ldots \ldots$ & ${ }^{4} D_{7 / 2}$ & $\ldots$ & $-1.13 \pm 0.07,3.7$ & $\ldots$ & $\ldots$ & $-1.03 \pm 0.07$ \\
\hline $3281.30 \ldots \ldots$ & ${ }^{6} D_{5 / 2}$ & $-2.69 \pm 0.04,4.1$ & $\ldots$ & $-2.69 \pm 0.04,4.1^{\mathrm{a}}$ & $\ldots$ & $-2.65 \pm 0.04$ \\
\hline $3285.41 \ldots \ldots$ & ${ }^{6} D_{1 / 2}$ & $-2.87 \pm 0.04,3.9$ & $-2.12 \pm 0.06,4.0$ & $\ldots$ & $\ldots$ & $-2.82 \pm 0.04$ \\
\hline $3295.81 \ldots \ldots$ & ${ }^{6} D_{3 / 2}$ & $-2.90 \pm 0.04,4.0$ & $\ldots$ & $-2.90 \pm 0.04,4.0^{\mathrm{a}}$ & $\ldots$ & $-2.87 \pm 0.04$ \\
\hline $3302.86 \ldots \ldots$ & ${ }^{6} D_{7 / 2}^{3 / 2}$ & $-3.51 \pm 0.07,4.2$ & $\cdots$ & $\ldots$ & $\cdots$ & $-3.45 \pm 0.07$ \\
\hline $3303.47 \ldots \ldots$ & ${ }^{6} D_{1 / 2}$ & $-2.70 \pm 0.11,3.9$ & $\ldots$ & $\ldots$ & $\ldots$ & $-2.68 \pm 0.11$ \\
\hline $4173.47 \ldots \ldots$ & ${ }^{4} D_{5 / 2}$ & $-2.18 \pm 0.07,3.2$ & $\ldots$ & $\ldots$ & $-2.77 \pm 0.14,3.4$ & $-2.50 \pm 0.08$ \\
\hline $4178.87 \ldots \ldots$ & ${ }^{4} F_{7 / 2}$ & $-2.48 \pm 0.11,3.9$ & $\ldots$ & $\ldots$ & $\ldots$ & $-2.45 \pm 0.11$ \\
\hline $4233.17 \ldots \ldots$ & ${ }^{4} D_{7 / 2}$ & $-1.91 \pm 0.07,3.7$ & $-2.00 \pm 0.11,3.7$ & $-1.91 \pm 0.07,3.7^{\mathrm{a}}$ & $\ldots$ & $-1.84 \pm 0.06$ \\
\hline $4303.17 \ldots \ldots$ & ${ }^{4} D_{3 / 2}$ & $-2.49 \pm 0.07,3.2$ & $\ldots$ & $-2.65 \pm 0.07,3.2$ & $\ldots$ & $-2.61 \pm 0.07$ \\
\hline $4351.76 \ldots \ldots$ & ${ }^{4} D_{5 / 2}$ & $-2.10 \pm 0.07,3.2$ & $\ldots$ & $-2.10 \pm 0.07,3.2^{\mathrm{a}}$ & $-1.99 \pm 0.08,3.4$ & $-2.03 \pm 0.05$ \\
\hline $4385.39 \ldots \ldots$ & ${ }^{4} D_{1 / 2}$ & $\ldots$ & $\ldots$ & $\ldots$ & $-2.75 \pm 0.06,3.4$ & $-2.69 \pm 0.06$ \\
\hline $4491.40 \ldots \ldots$ & ${ }^{4} F_{3 / 2}$ & $-2.70 \pm 0.11,4.1$ & $\ldots$ & $\ldots$ & $\ldots$ & $-2.66 \pm 0.11$ \\
\hline $4508.29 \ldots \ldots$ & ${ }^{4} D_{1 / 2}^{3 / 2}$ & $\ldots$ & $\ldots$ & $\ldots$ & $-2.58 \pm 0.09,3.4$ & $-2.52 \pm 0.09$ \\
\hline $4515.33 \ldots \ldots$ & ${ }^{4} F_{5 / 2}$ & $-2.41 \pm 0.11,4.0$ & $\ldots$ & $\ldots$ & $\ldots$ & $-2.36 \pm 0.11$ \\
\hline $4522.62 \ldots \ldots$ & ${ }^{4} D_{3 / 2}$ & $-2.03 \pm 0.11,3.2$ & $\ldots$ & $\ldots$ & $\ldots$ & $-1.99 \pm 0.11$ \\
\hline $4549.46 \ldots \ldots$ & ${ }^{4} D_{5 / 2}^{3 / 2}$ & $-1.75 \pm 0.07,3.2$ & $\cdots$ & $\cdots$ & $-2.33 \pm 0.30,3.4$ & $-1.87 \pm 0.08$ \\
\hline $4555.88 \ldots \ldots$ & ${ }^{4} F_{7 / 2}^{J / 2}$ & $-2.29 \pm 0.11,3.9$ & $\ldots$ & $\ldots$ & $\ldots$ & $-2.26 \pm 0.11$ \\
\hline $4576.33 \ldots \ldots$ & ${ }^{4} D_{5 / 2}$ & $\ldots$ & $\ldots$ & $-2.94 \pm 0.09,3.2$ & $-2.97 \pm 0.16,3.4$ & $-2.91 \pm 0.07$ \\
\hline $4582.83 \ldots \ldots$ & ${ }^{4} F_{7 / 2}$ & $-3.10 \pm 0.11,3.9$ & $\ldots$ & $\ldots$ & $\ldots$ & $-3.07 \pm 0.11$ \\
\hline $4583.83 \ldots \ldots$ & ${ }^{4} D_{7 / 2}$ & $-1.84 \pm 0.07,3.7$ & $-2.02 \pm 0.22,3.7$ & $\ldots$ & $\ldots$ & $-1.77 \pm 0.07$ \\
\hline $4620.51 \ldots \ldots$ & ${ }^{4} \mathrm{D}_{7 / 2}$ & $\ldots$ & $\ldots$ & $-3.29 \pm 0.08,3.7$ & $\ldots$ & $-3.19 \pm 0.07$ \\
\hline $4629.34 \ldots \ldots$ & ${ }^{4} F_{9 / 2}$ & $-2.33 \pm 0.11,4.3$ & $\ldots$ & $\ldots$ & $\ldots$ & $-2.28 \pm 0.11$ \\
\hline $4656.97 \ldots \ldots$ & ${ }^{4} D_{5 / 2}$ & $\ldots$ & $\ldots$ & $-3.61 \pm 0.10,3.2$ & $\ldots$ & $-3.58 \pm 0.08$ \\
\hline $4923.92 \ldots \ldots$ & ${ }^{6} P_{3 / 2}$ & $-1.24 \pm 0.11,4.0$ & $-1.32 \pm 0.11,4.0$ & $\ldots$ & $\ldots$ & $-1.26 \pm 0.08$ \\
\hline $5169.03 \ldots \ldots$ & ${ }^{6} P_{7 / 2}^{3 / 2}$ & $-0.87 \pm 0.11,3.8$ & $\ldots$ & $\ldots$ & $\ldots$ & $-0.86 \pm 0.11$ \\
\hline $5197.57 \ldots \ldots$ & ${ }^{4} F_{3 / 2}$ & $-2.10 \pm 0.11,4.1$ & $\ldots$ & $\ldots$ & $\ldots$ & $-2.06 \pm 0.11$ \\
\hline $5234.66 \ldots \ldots$ & ${ }^{4} F_{5 / 2}^{3 / 2}$ & $-2.05 \pm 0.11,4.0$ & $\cdots$ & $-2.27 \pm 0.10,4.0^{\mathrm{b}}$ & $\cdots$ & $-2.23 \pm 0.08$ \\
\hline $5262.47 \ldots \ldots$ & ${ }^{4} D_{5 / 2}^{5 / 2}$ & $\ldots$ & $\cdots$ & $-3.06 \pm 0.10,3.2^{\mathrm{b}}$ & $\cdots$ & $-3.03 \pm 0.10$ \\
\hline $5264.79 \ldots \ldots$ & ${ }^{4} D_{3 / 2}$ & $\ldots$ & $\ldots$ & $-3.27 \pm 0.08,3.2$ & $\ldots$ & $-3.23 \pm 0.05$ \\
\hline $5276.00 \ldots \ldots$ & ${ }^{4} F_{7 / 2}^{3 / 2}$ & $-1.94 \pm 0.11,3.9$ & $\ldots$ & $\ldots$ & $\ldots$ & $-1.91 \pm 0.11$ \\
\hline $5316.62 \ldots \ldots$ & ${ }^{4} F_{9 / 2}$ & $-1.85 \pm 0.11,4.3$ & $\ldots$ & $\ldots$ & $\cdots$ & $-1.85 \pm 0.11$ \\
\hline $5316.78 \ldots \ldots$ & ${ }^{4} D_{5 / 2}^{9 / 2}$ & $\ldots$ & $\ldots$ & $\ldots$ & $-2.80 \pm 0.12,3.4$ & $-2.74 \pm 0.12$ \\
\hline $5325.55 \ldots \ldots$ & ${ }^{4} F_{7 / 2}$ & $-2.60 \pm 0.11,3.9$ & $\ldots$ & $\ldots$ & $\ldots$ & $-2.57 \pm 0.11^{\mathrm{c}}$ \\
\hline $5414.08 \ldots \ldots$ & ${ }^{4} D_{7 / 2}$ & $\ldots$ & $\ldots$ & $-3.58 \pm 0.09,3.7$ & $\ldots$ & $-3.48 \pm 0.08$ \\
\hline $5525.13 \ldots \ldots$ & ${ }^{4} D_{7 / 2}$ & $\ldots$ & $\ldots$ & $-4.04 \pm 0.11,3.7$ & $\ldots$ & $-3.94 \pm 0.09$ \\
\hline $5607.12 \ldots \ldots$ & ${ }^{6} D_{7 / 2}$ & $\ldots$ & $\ldots$ & $-3.83 \pm 0.07,4.2$ & $\ldots$ & $-3.77 \pm 0.07$ \\
\hline $5627.49 \ldots \ldots$ & ${ }^{4} F_{5 / 2}$ & $\ldots$ & $\ldots$ & $-4.14 \pm 0.09,4.0$ & $\ldots$ & $-4.10 \pm 0.07$ \\
\hline $6369.42 \ldots \ldots$ & ${ }^{6} D_{3 / 2}$ & $\ldots$ & $\ldots$ & $-3.50 \pm 0.12,4.0$ & $\ldots$ & $-3.47 \pm 0.12$ \\
\hline $6432.67 \ldots \ldots$ & ${ }^{6} D_{5 / 2}^{3 / 2}$ & $\cdots$ & $\cdots$ & $-3.55 \pm 0.08,4.1$ & $\cdots$ & $-3.51 \pm 0.06$ \\
\hline $6516.07 \ldots \ldots$ & ${ }^{6} D_{7 / 2}^{5 / 2}$ & $\cdots$ & $\cdots$ & $-3.44 \pm 0.07,4.2$ & $\cdots$ & $-3.38 \pm 0.05$ \\
\hline $7222.39 \ldots \ldots$ & ${ }^{4} D_{1 / 2}$ & $\ldots$ & $\ldots$ & $\ldots$ & $-3.43 \pm 0.07,3.4$ & $-3.37 \pm 0.07$ \\
\hline $7224.49 \ldots \ldots$ & ${ }^{4} D_{1 / 2}$ & $\ldots$ & $\ldots$ & $\ldots$ & $-3.35 \pm 0.06,3.4$ & $-3.29 \pm 0.06$ \\
\hline $7449.34 \ldots \ldots$ & ${ }^{4} D_{5 / 2}$ & $\ldots$ & $\ldots$ & $-3.10 \pm 0.11,3.2$ & $\ldots$ & $-3.07 \pm 0.09$ \\
\hline $7515.83 \ldots \ldots$ & ${ }^{4} D_{5 / 2}^{5 / 2}$ & $\ldots$ & $\ldots$ & $-3.41 \pm 0.12,3.2$ & $-3.53 \pm 0.08,3.4$ & $-3.45 \pm 0.07$ \\
\hline $7711.73 \ldots \ldots$ & ${ }^{4} D_{7 / 2}$ & $\ldots$ & $\ldots$ & $-2.55 \pm 0.08,3.7$ & $\ldots$ & $-2.45 \pm 0.07$ \\
\hline
\end{tabular}

NoTE.-Lifetime values (in ns) follow $\log g$ f values and errors (in dex).

${ }^{a}$ Heise \& Kock (1990) lines taken as reference from Kroll \& Kock (1987).

b Line classified as a possible blend or a misidentification by Heise \& Kock (1990).

${ }^{c}$ This line has $\log g f=-3.2$ as computed by Kurucz. Only with a value similar to that can we reconcile the iron abundance from this line with that from the other $\mathrm{Fe}$ in lines. 


\section{REFERENCES}

Allende Prieto, C., Barklem, P. S., Asplund, M., \& Ruiz Cobo, B. 2001, ApJ, 558, 830

Allende Prieto, C., \& García Lopez, R. J. 1998, A\&AS, 131, 431

Allende Prieto, C., Garcia Lopez, R. J., Lambert, D. L., \& Gustafsson, B. 1999a, ApJ, 526, 991 1999 b, ApJ, 527, 879

Allende Prieto, C., García López, R. J., \& Trujillo Bueno, J. 1997, ApJ, 483, 941

Allende Prieto, C., \& Lambert, D. L. 1999, A\&A, 352, 555

Anstee, S. D., \& O'Mara, B. J. 1991, MNRAS, 253, 549

Asplund, M. 2000, A\&A, 359, 755

Asplund, M., \& García Pérez, A. E. 2001, A\&A, 372, 601

Asplund, M., Gustafsson, B., Kiselman, D., \& Eriksson, K. 1997, A\&A, 318,521

Asplund, M., Ludwig, H.-G., Nordlund, Å., \& Stein, R. F. 2000a, A\&A, 359, 669

Asplund, M., Nordlund, Å., Trampedach, R., Allende Prieto, C., \& Stein, R. F. 2000b, A\&A, 359, 729

Asplund, M., Nordlund, A., Trampedach, R., \& Stein, R. F. 1999, A\&A, 346, L17 2000c, A\&A, 359, 743

Atroshchenko, I. N., \& Gadun, A. S. 1994, A\&A, 291, 635

Barklem, P. S., \& O'Mara, B. J. 1997, MNRAS, 290, 102

Barklem, P. S., O'Mara, B. J., \& Ross, J. E. 1998, MNRAS, 296, 1057

Barklem, P. S., Piskunov, N., \& O’Mara, B. J. 2000, A\&A, 363, 1091

Benz, W., \& Mayor, M. 1984, A\&A, 138, 183

Bertelli, G., Bressan, A., Chiosi, C., Fagotto, F., \& Nasi, E. 1994, A\&AS, 106,275

Biemont, E., Baudoux, M., Kurucz, R. L., Ansbacher, W., \& Pinnington, E. H. 1991, A\&A, 249, 539

Blackwell, D. E., Booth, A. J., Haddock, D. J., Petford, A. D., \& Leggett, S. K. 1986, MNRAS, 220, 549

Bruls, J. H. M. J., \& Rutten, R. J. 1992, A\&A, 265, 257

Chollet, F., \& Sinceac, V. 1999, A\&AS, 139, 219

Claverie, A., Isaak, G. R., McLeod, C. P., van der Raay, H. B., \& Cortes, T. R. 1979, Nature, 282, 591

di Benedetto, G. P. 1998, A\&A, 339, 858

Dravins, D. 1987, A\&A, 172, 211 1990, A\&A, 228, 218 1999, in ASP Conf. Ser. 185, Precise Stellar Radial Velocities, ed.

J. B. Hearnshaw \& C. D. Scarfe (San Francisco: ASP), 268

Dravins, D., Larsson, B., \& Nordlund, A. 1986, A\&A, 158, 83

Dravins, D., Lindegren, L., \& Nordlund, Å. 1981, A\&A, 96, 345

Dravins, D., \& Nordlund, Å. 1990, A\&A, 228, 184

Fekel, F. C. 1997, PASP, 109, 514

Fossat, E., \& Ricort, G. 1975, A\&A, 43, 243

Fuhrmann, K., Pfeiffer, M., Frank, C., Reetz, J., \& Gehren, T. 1997, A\&A, 323, 909

Girard, T. M., et al. 2000, AJ, 119, 2428 2000, AJ, 119, 2428

Giridhar, S., \& Ferro, A. A. 1995, Rev. Mexicana Astron. Astrofis., 31, 23

Gray, D. F. 1981a, ApJ, 251, 152 .1981b, ApJ, 251, 583
Gray, D. F. 1982, ApJ, 255, 200

Gray, D. F., \& Nagel, T. 1989, ApJ, 341, 421

Gray, D. F., \& Toner, C. G. 1986, PASP, 98, 499

Grevesse, N., \& Sauval, A. J. 1998, Space Sci. Rev., 85, 161

Griffin, R., \& Griffin, R. 1979, A Photometric Atlas of the Spectrum of Procyon $\lambda \lambda 3140-7470 \AA$ (Cambridge: Institute of Astronomy, Observatories)

Guo, B., Ansbacher, W., Pinnington, E. H., Ji, Q., \& Berends, R. W. 1992, Phys. Rev. A, 46, 641

Gustafsson, B., Bell, R. A., Eriksson, K., \& Nordlund, Å. 1975, A\&A, 42, 407

Hamilton, D., \& Lester, J. B. 1999, PASP, 111, 1132

Hanbury Brown, R., Davis, J., \& Allen, L. R. 1974, MNRAS, 167, 121

Hannaford, P., Lowe, R. M., Grevesse, N., \& Noels, A. 1992, A\&A, 259, 301

Heise, C., \& Kock, M. 1990, A\&A, 230, 244

Irwin, A. W., Fletcher, J. M., Yang, S. L. S., Walker, G. A. H., \& Goodenough, C. 1992, PASP, 104, 489

Kiselman, D., \& Nordlund, A. 1995, A\&A, 302, 578

Kroll, S., \& Kock, M. 1987, A\&AS, 67, 225

Kulander, J. L., \& Jefferies, J. T. 1966, ApJ, 146, 194

Kupka, F., Piskunov, N., Ryabchikova, T. A., Stempels, H. C., \& Weiss, W. W. 1999, A\&AS, 138, 119

Kurucz, R. L., Furenlid, I., \& Brault, J. 1984, National Solar Observatory Atlas, Sunspot (New Mexico: NSO)

Lambert, D. L., Heath, J. E., Lemke, M., \& Drake, J. 1996, ApJS, 103, 183

Lindegren, L., Dravins, D., \& Madsen, S. 1999, in ASP Conf. Ser. 185, Precise Stellar Radial Velocities, IAU Colloq. 170, ed. J. B. Hearnshaw \& C. D. Scarfe (San Francisco: ASP), 73

Martić, M., et al. 1999, A\&A, 351,993

Mihalas, D., Dappen, W., \& Hummer, D. G. 1988, ApJ, 331, 815

Mozurkewich, D., et al. 1991, AJ, 101, 2207

Nave, G., Johansson, S., Learner, R. C. M., Thorne, A. P., \& Brault, J. W. 1994, ApJS, 94, 221

Nordlund, A. 1982, A\&A, 107, 1

Nordlund, A., \& Dravins, D. 1990, A\&A, 228, 155

O'Brian, T. R., Wickliffe, M. E., Lawler, J. E., Whaling, W., \& Brault, J. W. 1991, J. Opt. Soc. Am. B, 8, 1185

Pauls, U., Grevesse, N., \& Huber, M. C. E. 1990, A\&A, 231, 536

Provencal, J. L., Shipman, H. L., Wesemael, F., Bergeron, P., Bond, H. E., Liebert, J., \& Sion, E. M. 1997, ApJ, 480, 777

Rice, J. B., \& Wehlau, W. H. 1984, ApJ, 278, 721

Schnabel, R., Kock, M., \& Holweger, H. 1999, A\&A, 342, 610

Shchukina, N., \& Trujillo Bueno, J. 2001, ApJ, 550, 970

Sneden, C. 1973, Ph.D. thesis, Univ. Texas at Austin

Stein, R. F., \& Nordlund, Å. 1998, ApJ, 499, 914

Thévenin, F. 1989, A\&AS, 77, 137 1990, A\&AS, 82, 179

Trampedach, R. 1997, M.S. thesis, Univ. Aarhus

Tull, R. G., MacQueen, P. J., Sneden, C., \& Lambert, D. L. 1995, PASP, 107,251

Unsöld, A. 1955, Physik der Sternatmosphären (Berlin: Springer) 